\title{
Change in interface magnetism of an exchange-coupled system due to the presence of nonmagnetic spacers
}

\author{
Amitesh Paul, ${ }^{1, *}$ N. Paul, ${ }^{2}$ Jaru Jutimoosik, ${ }^{3}$ Rattikorn Yimnirun, ${ }^{3}$ Saroj Rujirawat, ${ }^{3}$ Britta Höpfner, ${ }^{2}$ Iver Lauermann, ${ }^{2}$ \\ M. Lux-Steiner, ${ }^{2}$ Stefan Mattauch, ${ }^{4}$ and Peter Böni ${ }^{1}$ \\ ${ }^{1}$ Technische Universität München, Physik Department E21, Lehrstuhl für Neutronenstreuung, James-Franck-Strasse 1, \\ D-85748 Garching b. München, Germany \\ ${ }^{2}$ Helmholtz-Zentrum Berlin für Materialien und Energie GmbH, Hahn-Meitner-Platz 1, D-14109 Berlin, Germany \\ ${ }^{3}$ School of Physics, Institute of Science, Suranaree University of Technology, Nakhon Ratchasima, and Thailand Center of Excellence in \\ Physics (ThEP Center), Commission on Higher Education, Bangkok, Thailand \\ ${ }^{4}$ Jülich Centre for Neutron Science Forschungszentrum Jülich GmbH, Außenstelle am FRM-II c/o TU München, Lichtenbergstraße 1, \\ D-85747 Garching b. München, Germany
}

(Received 27 April 2012; revised manuscript received 3 September 2012; published 28 January 2013)

\begin{abstract}
We report on the effect of nonmagnetic spacer layers on the interface magnetism and the exchange bias in the archetypical $[\mathrm{Co} / \mathrm{CoO}]_{16}$ system. The separation of the magnetic bilayers by Au layers with various thicknesses $d_{\mathrm{Au}} \geqslant 25 \mathrm{~nm}$ leads to a threefold increase of the exchange bias field $\left(H_{\mathrm{eb}}\right)$. Reflectometry with polarized neutrons does not reveal any appreciable change in the domain population. This result is in agreement with the observation that the granular microstructure within the [Co/CoO] bilayers is independent of $d_{\mathrm{Au}}$. The significant reduction of the magnetic moments in the Co layers can be attributed to interfacial disorder at the Co-Au interfaces. Element-specific x-ray absorption spectroscopy attributes part of the enhancement of $H_{\mathrm{eb}}$ to the formation of $\mathrm{Co}_{3} \mathrm{O}_{4}$ in the $[\mathrm{Co} / \mathrm{CoO}]$ bilayers within the multilayers. A considerable proportion of the increase of $H_{\mathrm{eb}}$ can be attributed to the loss of magnetization at each of the Co-Au interfaces with increasing $d_{\mathrm{Au}}$. We propose that the interfacial magnetism of ferro- and antiferromagnetic layers can be significantly altered by means of metallic spacer layers thus affecting the exchange bias significantly. This study shows that the magnetism in magnetic multilayers can be engineered by nonmagnetic spacer layers without involving the microstructure of the individual layers.
\end{abstract}

DOI: 10.1103/PhysRevB.87.014431

PACS number(s): 75.70.Cn, 75.60.Jk

\section{INTRODUCTION}

As new magnetic hard-disk-drive products are designed for higher storage densities in magnetic recording materials, the "superparamagnetic effect" has become increasingly important. ${ }^{1}$ As the grains become smaller $(50-100 \mathrm{~nm})$, due to thermally activated fluctuations, the magnetization of the grains may become unstable. One approach to delay superparamagnetism is to increase the magnetic anisotropy or the unidirectional anisotropy.

The exchange bias phenomenon can be described as a form of a unidirectional magnetic anisotropy that arises due to the interfacial exchange coupling between a ferromagnet (FM) and an antiferromagnet (AF) and can effectively delay the superparamagnetic limit. ${ }^{2}$ In most usual cases, the AF ordering temperature is lower than that of the FM, below which one observes a horizontal shift of the hysteresis loop. However, temperature-dependent competition between interfacial exchange and $\mathrm{AF}$ anisotropy energies can result in bias fields even for materials with higher $\mathrm{AF}$ ordering temperature. ${ }^{3}$ Conventionally, a cooling field $\left(H_{\mathrm{FC}}\right)$ provides the unidirectional anisotropy while the shift is observed opposite to the applied field $\left(H_{\mathrm{a}}\right)$ direction. Over the last decade many salient features of the exchange bias effect have been clarified. It turns out that only a very small percentage of moments at the AF interface are pinned while the rest of the moments rotate rigidly with the FM. It also turns out that it is energetically favorable to form domains in the antiferromagnet. They account for the lowering of the energy cost associated with the reversal of the FM that determines the strength of the bias field $\left(H_{\mathrm{eb}}\right)^{4-6}$ Exchange bias is also associated with many salient features such as coercivity enhancement, ${ }^{7,8}$ asymmetric hysteresis loops, ${ }^{9,10}$ and training effects. ${ }^{11}$

One of the interesting problems in multilayer physics is the influence of the interface between the magnetic film and the nonmagnetic spacer on kinetic, magnetic, and magnetooptical properties of thin-film systems. Information concerning effects of (a) an underlayer grain morphology and a grain crystallographic orientation (texture of the grains) on magnetic properties, ${ }^{12}$ (b) induced magnetic moments via $s$ - $d$ hybridization, ${ }^{13}$ (c) interface alloying, ${ }^{14}$ and (d) canted magnetic structure are intrinsic to interfaces between magnetic-nonmagnetic magnetic layers. ${ }^{15}$ These are highly relevant to systems that are used as magnetic field sensors, read heads, or memory devices.

It may be noted that exchange bias systems are often coated with a Au film, in order to protect them against further oxidation. ${ }^{16}$ Moreover, Au is often used as metallic leads for spin-valve structures. Thus the $\mathrm{Au} / \mathrm{FM}$ (or $\mathrm{AF}$ ) interfaces and their effect on exchange bias cannot be ignored. In general, the introduction of a nonmagnetic (NM) metallic spacer such as $\mathrm{Cu}, \mathrm{Ag}$, or Au between the FM and FM/AF layers modifies the interface coupling between them. Therefore it is of great interest to obtain information about the spin directions in the vicinity of the interfaces. This aspect, however, remains largely unexplored. In fact, there are no studies on the impact of the 
$\mathrm{AF} / \mathrm{Au}$ or $\mathrm{FM} / \mathrm{Au}$ interface magnetism including the effects of roughness and interdiffusion on the exchange bias phenomena. The aim of this study is, therefore, to systematically investigate the magnetisation of exchange coupled bilayers of $\mathrm{Co} / \mathrm{CoO}$ that are separated by nonmagnetic Au spacer layers.

Contrary to the expectations, we show here that the exchange bias field increases gradually with increasing thickness of the Au spacer layer. As expected, the magnetization reversal mechanism remains asymmetric for the two branches of the hysteresis loops, however, it shows significantly increased coerciveness along both branches with increasing thickness of the Au layers. These effects occur despite the fact that the diameter of the magnetic grains attains a similar size as the $\mathrm{Au}$ spacer thickness and that the FM domains show no significant variation in their size. It appears that the impact of the metallic $\mathrm{Au}$ spacer adjacent to an AF or FM is very significant for exchange bias systems in general as it can alter the interfacial magnetism.

\section{SAMPLES AND MEASUREMENTS}

\section{A. Sample preparation}

Over the years, $\mathrm{Co} / \mathrm{CoO}$ has served as a prototypical exchange bias system, even though it is not actually technologically practical. In fact, very recent extensive investigations are on the same AF/FM combination. ${ }^{16-19}$ It is ideal for investigation due to its large biasing field, ${ }^{6}$ very distinct asymmetry of magnetization reversal, ${ }^{5}$ large enough training effects, ${ }^{11}$ and most interestingly, the AF moment configuration can be frozen-in in a variety of ways during the process of field cooling ${ }^{20}$ without affecting the overall structure as the AF ordering temperature is far below room temperature (negligible interdiffusion at the interfaces).

We have investigated multilayers of the composition $\mathrm{SiO}_{2} /$ $[\mathrm{Co}(11.0 \mathrm{~nm}) / \mathrm{CoO}(5.0 \mathrm{~nm}) / \mathrm{Au}(25,30,50 \mathrm{~nm})]_{\mathrm{N}=16}$ and compare them with $\mathrm{SiO}_{2} /[\mathrm{Co}(11.0 \mathrm{~nm}) / \mathrm{CoO}(7.0 \mathrm{~nm})]_{\mathrm{N}=20} /$ $\mathrm{Au}(50 \mathrm{~nm})$. A schematic of the layer structure is shown in Fig. 1. During deposition, the Ar pressure in the magnetron sputtering chamber was $3 \times 10^{-3}$ mbar. The process was started at a base pressure of $1 \times 10^{-7}$ mbar. We employ an

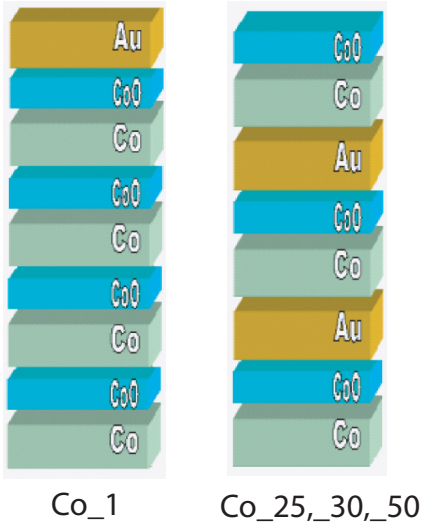

FIG. 1. (Color online) Schematic of the layer structures, namely, Co_1 having no spacer layer and Co_25, Co_30, and Co_50 having the bilayers separated by Au spacer layers. ultraviolet light assisted oxidation at an $\mathrm{O}_{2}$ pressure of 200 mbar at $50{ }^{\circ} \mathrm{C}$ for 1 hour. $^{21}$

\section{B. Measurement techniques}

\section{Magnetometery}

Conventional in-plane magnetization loops are measured using a superconducting quantum interference device (SQUID) MPMS and a physical property measurement system (PPMS) from Quantum design. We use a cooling field $H_{\mathrm{FC}}=$ $+4.0 \mathrm{kOe}$ within the sample plane for all specimens inducing an exchange bias as the system is cooled down to $10 \mathrm{~K}$.

\section{X-ray scattering and microscopy}

$\mathrm{X}$-ray diffraction patterns from the samples confirm the $[111]_{\text {fcc }}$ structure for the Au and Co layers. The microstructural characterization was performed using cross-sectional transmission electron microscopy (XTEM). Studies with transmission electron microscopy have been carried out on cross-sectional samples prepared by standard mechanical (diamond) polishing followed by $\mathrm{Ar}^{+}$ion milling at $4 \mathrm{kV}$ for about 1 hour. A conventional bright-field imaging mode was used.

\section{Polarized neutron scattering}

Polarized neutrons are an excellent probe for investigating the in and out of plane correlations of the ferromagnetic domains. Depth-sensitive polarized neutron scattering measurements are performed at the neutron reflectometer TREFF at FRM II using polarization analysis. The specular as well as the off-specular data were measured. The neutron wavelength was fixed at $\lambda=4.73 \AA$. Details on the technique and a corresponding review can be found elsewhere. ${ }^{23}$ In the experiment, four different cross sections are measured, namely, non-spin-flip (NSF) $\left(R_{++}\right.$and $\left.R_{--}\right)$and spin-flip (SF) $\left(R_{+-}\right.$ and $R_{-+}$) channels . Here, the subscripts + and - designate polarizations of the neutron beam parallel or antiparallel to the guide field, respectively. The specimens are field cooled in $H=4.0 \mathrm{kOe}$ to $10 \mathrm{~K}$ inside a cryostat at the instrument. The NSF intensities provide the amplitude of the projection of the magnetization along the polarization direction of the neutrons $\left(\mathrm{M}_{\|}\right)$, while the SF intensities provide information about the magnetization components perpendicular to the polarization direction $\left(\mathrm{M}_{\perp}\right)$. The latter contributions are exclusively of magnetic origin.

\section{X-ray absorption spectroscopy}

An increase in the bias field $H_{\mathrm{eb}}$ can originate from the formation of defects within the antiferromagnetic $\mathrm{Co}_{x} \mathrm{O}_{y}$ layer or from deviations in the stoichiometry during the course of the oxidation of $\mathrm{Co}$ to $\mathrm{CoO}^{21}$ leading to a stronger pinning of the domain walls at the defect sites thus resulting in an increase of $H_{\mathrm{eb}}{ }^{24}$ To verify the formation of such defect sites that can be inadvertently related to the degree of oxidation of the Co layer (few nanometers), it is necessary to investigate the proportion and stoichiometry of the CoO layers in the system. Such a detailed examination of the chemical species can be effectively done by x-ray absorption spectroscopy (XAS). 
TABLE I. Samples and their saturation magnetization and exchange energy. The bilayers Co/CoO of sample Co_1 are not separated by Au spacer layers.

\begin{tabular}{lccc}
\hline \hline Composition & Label & $\begin{array}{c}M_{\mathrm{FM}} \\
\left(\mathrm{emu} \mathrm{cm}{ }^{-3}\right)\end{array}$ & $\begin{array}{c}\text { Magnetic moment } \\
\mu_{\mathrm{B}} / \mathrm{Co}(\mathrm{FM})\end{array}$ \\
\hline$[\mathrm{Co}(11.0 \mathrm{~nm}) / \mathrm{CoO}(7.0 \mathrm{~nm})]_{20} / \mathrm{Au}(50 \mathrm{~nm})$ & $\mathrm{Co} \_1$ & $1694 \pm 100$ & $2.01 \pm 0.2$ \\
$\left.(\mathrm{erg} \mathrm{cm})^{-2}\right)$
\end{tabular}

XAS is generally used to obtain information about the local arrangement of atoms around the absorbing atoms. In particular, the X-ray absorption near-edge structure (XANES) region corresponds to the excitation of core electrons to unoccupied bound states or to low lying continuum states. It thus turns out that the angular momentum and site projected partial density of empty states, with some broadening, resemble the XANES absorption spectra.

The Co $K$-edge XANES measurements were performed in the fluorescent mode with a 13-component Ge detector at the x-ray absorption spectroscopy beamline (BL-8) of the Siam Photon Source (electron energy of $1.2 \mathrm{GeV}$, beam current 120-80 mA), Synchrotron Light Research Institute, Thailand. A double crystal monochromator Ge (220) was used to scan the energy of the synchrotron x-ray beam with energy steps of $0.30 \mathrm{eV}$.

Further, we performed Co $L_{2,3}$ edge XAS measurements on the specimens. The XAS spectra result from Co $2 p \longrightarrow$ $3 d$ dipole transitions $\left(2 p^{3 / 2}\right.$ and $2 p^{1 / 2}$ core-shell electrons to unoccupied $3 d$ orbitals). ${ }^{25}$ Comparing with the $a b$ initio calculations of the $L$-edge and $K$-edge structure of $\mathrm{Co}, \mathrm{CoO}$, and $\mathrm{Co}_{3} \mathrm{O}_{4}$, it is possible to identify the individual constituents of magnetic species in the system.

The absorption cross section is measured by collecting the energy selective fluorescence yield using a commercial XES300 spectrometer with an energy resolution of $0.89 \mathrm{eV}$ at the CISSY end station of the high-flux beamline U49/2-PGM1 installed at the Berliner Elektronenspeicherring Gesellschaft für Synchrotronstrahlung GmbH (BESSY). The photon energy is swept through the $L 3(778 \mathrm{eV})$ and $L 2(798 \mathrm{eV})$ edges of Co. The detector consists of a multichannel plate in conjunction with a resistive anode assembly. We integrate the $\mathrm{x}$-ray emission spectroscopic signal to get the florescence signal.

In principle, $\mathrm{X}$-ray magnetic dichroism (XMCD) can selectively probe the induced magnetic moment of $\mathrm{Au}$ in $\mathrm{Co} / \mathrm{Au}$ multilayers and separate it into spin and orbital terms. ${ }^{26}$ However, XMCD (sensitive to $p, d$, and $f$-electron polarization) is a surface sensitive technique as the probing depth in the soft $\mathrm{x}$-ray regime is $\sim 5.0 \mathrm{~nm}$ in the electron yield (EY) mode and $\sim 100 \mathrm{~nm}$ in the fluorescence yield (FY) mode. FY dichroism measurements are extremely sensitive to saturation and self-absorption effects, complicating the evaluation. Thus it is almost impossible to investigate the interface of an ML with a thicker spacer at deeply buried interfaces (as in the present case).

Alternatively, by using the low-temperature nuclear orientation (LTNO) technique, one can detect the average magnitude and alignment of the nuclear spins which can be due to the induced nuclear polarization in the nonmagnetic Au spacer $(s-$ moment polarization). ${ }^{15}$ Canting of the induced $\mathrm{Au}$ magnetic moments was found to originate at the $\mathrm{AF}(\mathrm{FM}) / \mathrm{Au} / \mathrm{AF}(\mathrm{FM})$ interface as well as canting of the Co moments (reducing the net moment of the uncompensated spins) was observed earlier in $\mathrm{AF} / \mathrm{Au} / \mathrm{FM}$ interfaces. However, a detailed inspection of the interface magnetization (depending upon the structure of the interface) was limited by the level of resolution available with the technique, and it also requires milli-Kelvin sample environment, which is not commonly available.

\section{RESULTS AND DISCUSSION}

\section{A. Magnetization}

The labeling of the samples along with the saturation magnetization per unit volume $\left(M_{\mathrm{FM}}\right)$ and the magnetic moment per Co (FM) atom is given in Table I. The exchange coupling energy ${ }^{27}$ per unit surface area is usually given by

$$
\begin{aligned}
E & =-J_{\mathrm{E}} M_{\mathrm{AF}} M_{\mathrm{FM}} t_{\mathrm{FM}} \cos \delta \\
& =-H_{\mathrm{eb}} M_{\mathrm{FM}} t_{\mathrm{FM}} \cos \delta .
\end{aligned}
$$

The unidirectional anisotropy energy is characterized by the exchange coupling constant $J_{\mathrm{E}}$. The unidirectional anisotropy $K_{\mathrm{ud}}$ is included in $J_{\mathrm{E}} M_{\mathrm{AF}} M_{\mathrm{FM}}$ in terms of the exchange field $H_{\mathrm{eb}}=J_{\mathrm{E}} M_{\mathrm{AF}}$. Here, $t_{\mathrm{FM}}$ is the thickness of the FM layer and $\delta$ is the angle between $M_{\mathrm{FM}}$ and the easy axis of the FM. $M_{F M}$ and $M_{\mathrm{AF}}$ are the respective magnetizations. We define the exchange bias shift $H_{\mathrm{eb}}=$ $\left(H_{\mathrm{C} 2}+H_{\mathrm{C} 1}\right) / 2$ and the coercive field $H_{\mathrm{C}}=\left(H_{\mathrm{C} 2}-H_{\mathrm{C} 1}\right) / 2$, where $H_{\mathrm{C} 1}$ and $H_{\mathrm{C} 2}$ are the coercive fields on the decreasing and increasing branches of the hysteresis loop, respectively. Also given in Table I are the exchange coupling energy $E$ as obtained from the respective FM layer thickness, the exchange bias field values and the saturation magnetizations for the MLs from the magnetization measurements.

\section{Hysteresis loops}

Figure 2(a) shows the hysteresis loops as measured with a SQUID for an in-plane cooling field and longitudinal magnetization measurements at $10 \mathrm{~K}$ for the sample Co_1. The results are reproduced from Ref. 32. For comparison, hysteresis loops for the samples Co_25, Co_30, and Co_50 are shown in Figs. 2(b)-2(d). Clearly seen is the usual asymmetry in the magnetization reversal and the disappearance of the asymmetry after the first field cycle. The room-temperature (RT) data [triangles in Figs. 2(b)-2(d)] show that the saturation field is around 100 Oe [for clarity, see the inset of Fig. 2(b)]. 

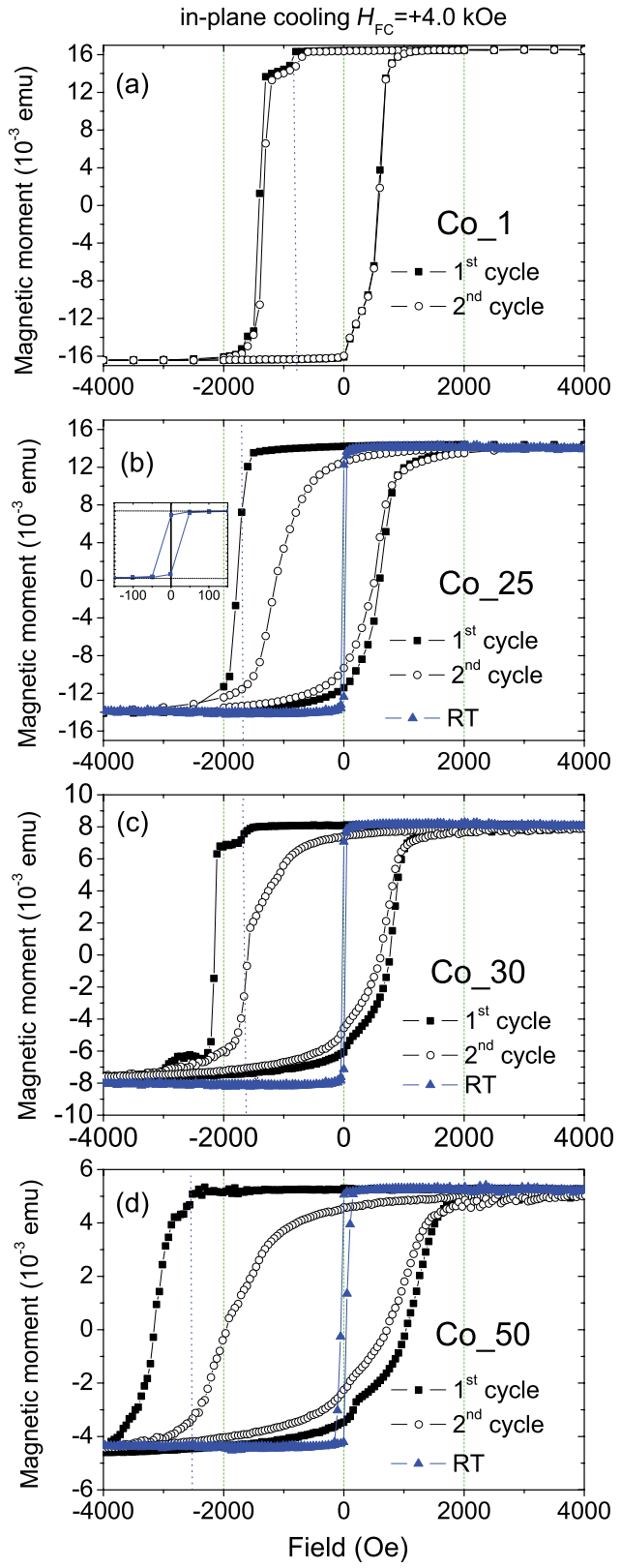

FIG. 2. (Color online) SQUID magnetization hysteresis loops for the (a) $[\mathrm{Co} / \mathrm{CoO}]_{20} \mathrm{ML}$ (from Ref. 32) and for $[\mathrm{Co} / \mathrm{CoO} / \mathrm{Au}]_{16} \mathrm{ML}$ for Au layer thicknesses of (b) $25 \mathrm{~nm}$ : Co_25 (c) $30 \mathrm{~nm}$ : Co_30 and (d) $50 \mathrm{~nm}$ : Co_50. The measurements are done at room temperature (triangles) and at $10 \mathrm{~K}$ (after cooling down in $H_{\mathrm{FC}}=+4 \mathrm{kOe}$ ). The inset in (b) shows the RT data in lower field values. The blue dotted lines indicate the switching field $H_{\mathrm{C}-\mathrm{O}}$ during the first field cycle. The thin solid lines are guides to the eye.

For Co_25 and Co_30, the coercive fields at RT and the exchange bias fields at $10 \mathrm{~K}$ are approximately 20 Oe and $\approx-580 \mathrm{Oe} / \approx-670 \mathrm{Oe}$, respectively. The corresponding RT data for Co_50 shows that the coercive field has increased to 40 Oe. Such a broadening of the hysteresis loop at RT can be generally attributed to defects within the magnetic layers. We point out that the exchange bias field along the cooling field axis is estimated to be around -1000 Oe for the $50 \mathrm{~nm}$ spacer ML, as compared to $\approx-400$ Oe for the ML specimen with no spacer.
The hysteresis loops in Fig. 2(a) show at least three kinks near $-780,-1400$, and around -1676 Oe along the decreasing branch. These kinks are an indication for $\mathrm{CoO}$ layers having different oxidation levels. Similar kinks can also be seen during the first cycle in Figs. 2(b)-2(d). The last switching fields show an increasing magnitude with increasing thickness of the spacer layer.

In a previous work, Paul et al. have found very similar characteristics while varying the oxidation conditions for the bottom and top Co layer in a $\mathrm{Co} / \mathrm{CoO} / \mathrm{Co}$ based spin-valve system. ${ }^{21}$ Note that similar subloops in oxidized Co dots were initially attributed to the effect of the aspect ratio for patterned samples ${ }^{28}$ even though they have been commonly observed in nonpatterned specimens as well. Intuitively, a varying stoichiometry of the $\mathrm{Co}_{x} \mathrm{O}_{y}$ layers, that may also depend on the number of bilayers, affects the strength of the exchange coupling between the AF and FM layers. Therefore an optimized stoichiometry can lead to an enhancement of the switching fields. Of course, the grain size may affect the switching fields as well.

The net magnetization in the Co_1 ML (for example) shows a decrease of 5\% after the first switching field along the decreasing branch of the hysteresis loop. This corresponds to $1 \mathrm{FM}$ layer out of the $20 \mathrm{FM}$ layers composing the ML, indicating that one of the 20 layers has already switched while the other 19 layers are on the verge of flipping. A similar argument can explain the magnetization data of the other samples, i.e., by a layer-by-layer flipping of the heterostructure.

\section{Magnetization versus temperature}

Figures 3(a) and 3(b) show the temperature dependence of the magnetization $\mathrm{M}$ of the samples Co_25 and Co_50 as
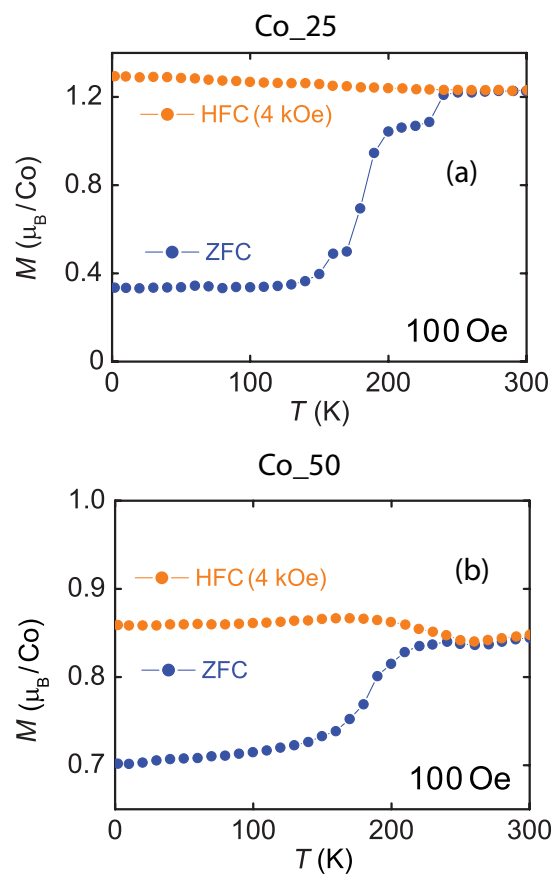

FIG. 3. (Color online) ZFC and HFC magnetizations as a function of increasing temperature $(T)$ in a small external field of $H=$ 100 Oe for (a) Co_25 ML and (b) Co_50 ML. 

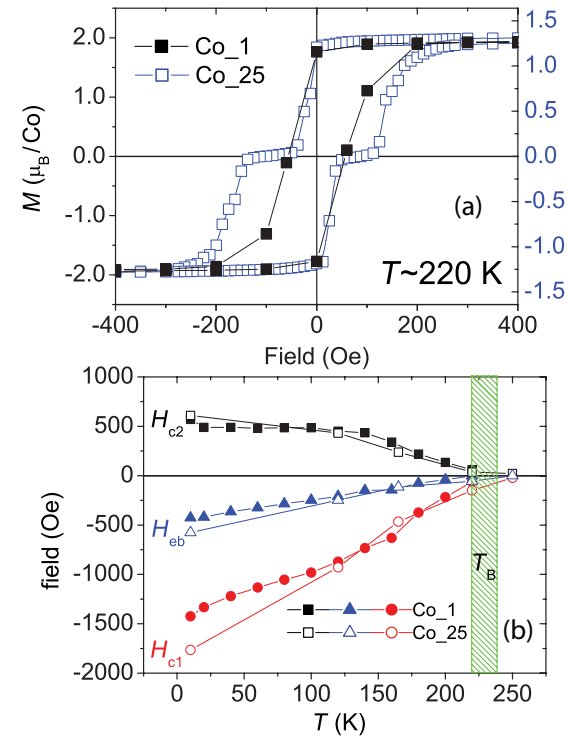

FIG. 4. (Color online) (a) SQUID magnetization hysteresis loops for the Co_1 and Co_25 MLs showing the sub-loops shifts. The measurements are done at $220 \mathrm{~K}$ (after cooling down in $H_{\mathrm{FC}}=$ $+4 \mathrm{kOe}$ ). (b) The temperature variation of the coercive fields and the exchange bias fields for the two MLs.

measured at 100 Oe using a PPMS. The merging point of the zero field cooled (ZFC) and field cooled (HFC, $H=4 \mathrm{kOe}$ ) data provide the blocking temperature $T_{\mathrm{B}}$ of the system. $T_{\mathrm{B}}$ characterizes the onset of instabilities of the AF as thermal excitations creep in. The similar $T_{\mathrm{B}}$ of both polycrystalline specimens indicates that their grain sizes are very similar. ${ }^{29}$ However, we find three distinct steps in Co_25 before the loops merge at $T_{\mathrm{B}}=240 \mathrm{~K}$. In Co_50, the steps are smeared out.

The samples show also a significant difference in the macroscopic magnetization during ZFC and HFC. The ZFC values at low $T$ are smaller for Co_25 than for Co_50. The HFC values are larger for Co_25 than for Co_50. These results indicate that the anisotropy in the Co_50 sample is larger.

\section{Initial domain configurations due to Au spacer}

Apart from the local inhomogeneities (roughness, defects) due to variations in the AF crystallite/grain sizes and concomitant domain size distribution, a distribution of local $T_{\mathrm{B}}$ is typically observed. It is well known that a thicker $\mathrm{AF}$ layer leads to an increased stability of the $\mathrm{AF}$ domains. ${ }^{29}$ Above a critical thickness (as in the present case), this may lead to splitting of the hysteresis loop into two subloops shifted in opposite directions when measured just around the blocking temperature. This subloop shifts and the temperature variation of the coercive fields can be seen in Figs. 4(a) and 4(b), respectively, for the Co_1 and Co_25 MLs. A marked difference is seen as we compare the MLs with and without the spacers. It is clear that the Co_1 ML does not show the subloop shifts. This clearly indicates that these shifts in the $\mathrm{Co} 25 \mathrm{ML}$ are due to the presence of $\mathrm{Co}-\mathrm{Au}$ and/or $\mathrm{CoO}-\mathrm{Au}$ interfaces as they together are responsible for a FM imprint onto an AF. Thus there is a particular difference in the initial AF-FM domain configurations in such systems, which can
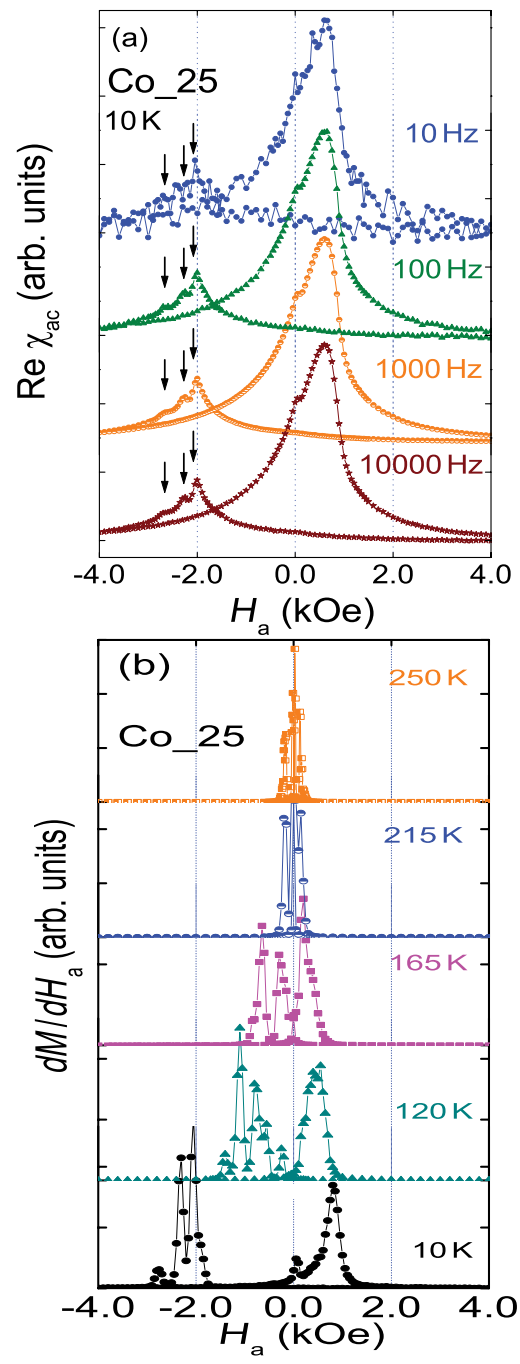

FIG. 5. (Color online) (a) Representative ac susceptibility measurements at different frequencies are shown for the Co_25 ML. (b) The field derivative of the magnetization as a function of field measured at various temperatures without applying an ac field is plotted for the Co_25 ML. They show the evolution of multiple switching with temperature along both branches of the hysteresis loop.

be a topic of future investigation. Usually, an imprint of the FM domain structure onto the AF during zero-field cooling procedure divides the $\mathrm{AF}$ into two types of regions locally oriented in opposite directions. ${ }^{6}$ Note that in the present case, the cooling field is above the saturation field of the FM and the FM orders before the AF. Here, a proportion of the AF spins/domains (affected by the thermal activation) is aligned by the cooling field, while another proportion remains unaligned. After field sweeping, this proportion gets realigned along the direction opposite to that initially set during the first field cooling.

\section{Susceptibility}

Susceptibility data of Co_25 are shown in Fig. 5(a). The in-phase susceptibility $\left(\operatorname{Re} \chi_{\mathrm{ac}}=d M / d H_{a}\right)$ data measured at $10 \mathrm{~K}$ and at a driving field of $10 \mathrm{Oe}(\mathrm{rms})$ after HFC from RT indicates also the occurrence of three reversal steps 


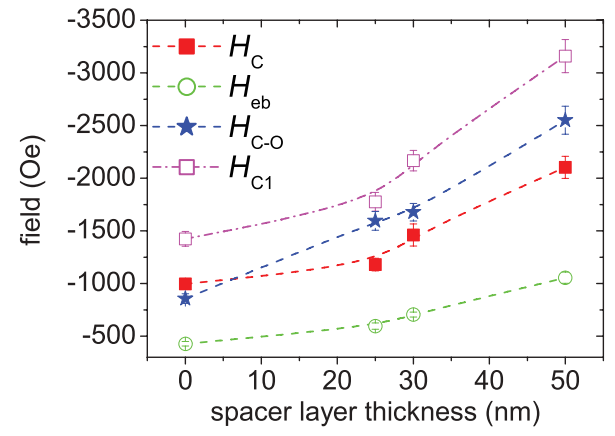

FIG. 6. (Color online) Dependence of $H_{\mathrm{eb}}$ and $H_{\mathrm{C}}$ on the thickness of the spacer layer as obtained from the SQUID magnetization hysteresis loops for $[\mathrm{Co} / \mathrm{CoO} / \mathrm{Au}]$ MLs. The coercive fields $H_{\mathrm{C} 1}$ and the first switching fields $H_{\mathrm{C}-\mathrm{O}}$ show an increase with increasing thickness. The dotted lines are guides to the eye.

(indicated by arrows) along the decreasing branch and two reversal steps along the increasing branch. The response from the samples hardly shows any frequency $(10 \mathrm{~Hz}-10 \mathrm{kHz})$ dependence. A much lower signal along the decreasing branch indicates that the domain dynamics along this branch is a slow process, at least slower than the response time corresponding to the $10 \mathrm{~Hz}$ of ac field. The reversal steps are more evident following the field derivative of the magnetization $d M / d H_{a}$ as a function of field in Fig. 5(b) following the data measured at various temperatures without an ac field. The evolution of the switching fields with temperature is consistent with the data in Fig. 3(a). The behavior for all other samples is very similar and is therefore not shown.

\section{5. $\mathrm{H}_{\mathrm{eb}}$ and $\mathrm{H}_{\mathrm{C}}$ with $\mathrm{Au}$ spacer thickness}

The plot of $H_{\mathrm{eb}}$ and $H_{\mathrm{C}}$ versus the spacer layer thickness in Fig. 6 shows an increasing magnitude with increasing spacer layer thickness. Also plotted is $H_{\mathrm{C} 1}$ and the first switching field $H_{\mathrm{C}-\mathrm{O}}$. While an increase in $H_{\mathrm{C}}$ can be associated with an increased number of nonpinned hysteretic AF grains, an increase in $H_{\mathrm{eb}}$ indicates an increase in the number of pinned domains or a stronger pinning by each domain in the polycrystalline specimens. Microstructural investigation could help in understanding such behavior further.

\section{B. Microstructure}

From the perspective of magneto-electronics, device characteristics are controlled by the magnetic evolution due to grain structure modulation. Each bit usually contains hundreds of grains. Magnetic recording relies on the statistical averaging over these grains to obtain a satisfactory signal to noise ratio. As the bit size continues to decrease, the grain size needs to be reduced too. The reduction can be achieved by controlling the surface properties of the coated and/or the noncoated substrate. However, eventually, the grains will become superparamagnetic. Thus a control over grain size is essential. Sputtered species have a high kinetic energy and surface mobility allowing rearrangements in the structure during film growth.

It was reported earlier that the exchange bias field can be increased with the number of bilayers with successive FM-AF interfaces. This is due to decreasing grain-size-mediated FM-AF exchange coupled domains stacked in successive layers with gradually smaller sizes. ${ }^{12}$ For polycrystalline specimens, within the random anisotropy model, the exchange interaction averages over the anisotropy of the individual grains. This would, in general, increase the effective exchange length. However, with an increasing number of smaller grains (with an increase in the number of bilayers), as the exchange length is reduced to the order of individual grain sizes $(\approx 50 \mathrm{~nm}$ in the present case), the random anisotropy model will break down. This will lead to the formation of individual exchange coupled grains - exchange coupled to the uncompensated AF moments preferably located at the grain boundaries. The spin alignment in individual FM domains is determined, domain by domain, by the spin directions in the AF grains. This is unlike the case of nanocrystallites where the grain sizes ( $\leqslant$ exchange length) concomitantly reduces the average anisotropy of the system and make them soft (lowered coercivity). ${ }^{30}$ In exchange-coupled systems, the rotatable anisotropy field value is proportional to the magnetization of the small AF grains, it increases with the exchange coupling strength, which in turn increases the coercivity. ${ }^{31}$ An increase in the coercivity with smaller AF grain sizes is basically due to an increase in the number of rotatable grains (proportional to the sum of the projections of these magnetizations along the bias direction).

Paul et al. ${ }^{32}$ have reported earlier on the magnetization reversal for (i) a continuous sequence of successive FM-AF layers (no spacer layers) and that for (ii) a sequence of FM-AF bilayers that are separated by a nonmagnetic spacer layer $\mathrm{Au})$. The main difference in their magnetization reversal mechanisms is the following: the separated multilayers (ML) showed a usual asymmetric reversal — a nonuniform (domain wall motion and domain nucleation) reversal for the decreasing branch $\left(H_{\mathrm{FC}}\right.$ anti- $\left.\| H_{\mathrm{a}}\right)$ of the hysteresis loop and a uniform (coherent rotation) reversal for the increasing branch $\left(H_{\mathrm{FC}} \| H_{\mathrm{a}}\right)$. In contrast, the continuous multilayer showed symmetric and sequential reversal (nonuniform) for both branches of the hysteresis loop.

In this regard, it is interesting to note that in contrast to the unlike case of a continuous ML (case (i) above), in a sequence of bilayers $\mathrm{Co}-\mathrm{CoO}$ that are interrupted by the presence of thick $\mathrm{Au}$ layers, the evolution of the grains may be interrupted depending on the thickness of the Au spacer layer, as the grain size is limited by the layer thickness. ${ }^{33}$ For a thick enough Au layer, the grain structure of the underlayer is not propagated to the next Co layer. This is similar to a decoupling of the intergranular interactions. ${ }^{34}$ It is therefore unlikely that the thickness of the nonmagnetic spacer will influence the magnetic grains as they are all nucleated on a similar spacer layer. Therefore one may speculate that the magnetic behavior does not change with an increasing thickness of the spacer layer. The aspect of grain structure evolution can be verified by cross-sectional TEM.

Figure 7 shows XTEM micrographs depicting repetitions of three layered structures with sharp interfaces for the MLs with (a) 25- and (b) 50-nm of spacer thickness. The thickness of the individual layers is in agreement with the nominal thickness. Magnifications of a trilayer interface show the existence of columnar grains with a width of $\approx 25 \mathrm{~nm}$ and $\approx 50 \mathrm{~nm}$ for the $25-\mathrm{nm}$ and $50-\mathrm{nm}$ sample, respectively. 

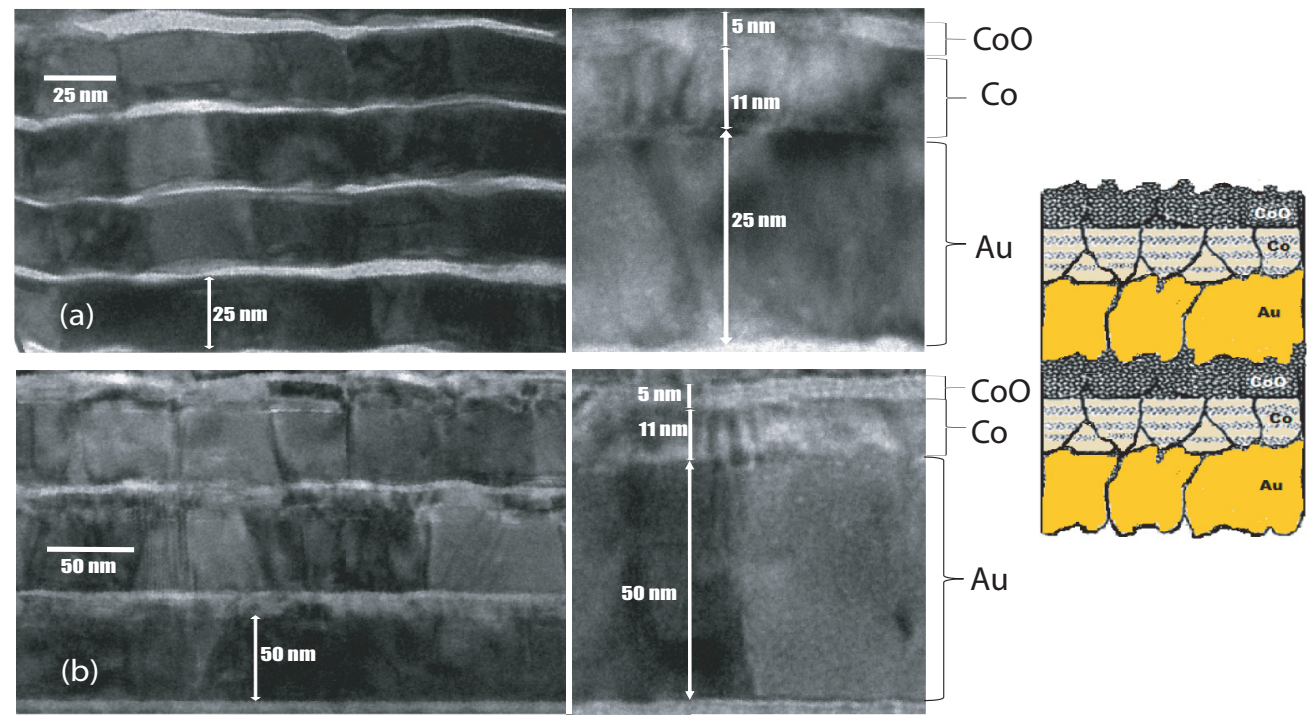

FIG. 7. (Color online) XTEM micrographs of $[\mathrm{Co} / \mathrm{CoO} / \mathrm{Au}]_{16} \mathrm{ML}$ for Au layer thicknesses of (a) 25 and (b) $50 \mathrm{~nm}$. Vertically correlated $\mathrm{Au}$ grains are visible for both MLs. There are no visible differences for the Co-CoO grains, which are basically unaffected by the size of the underlying Au grains. A schematic of the granular layer structure is shown alongside.

Note that the almost square-shaped Au grains are vertically correlated. The results confirm the common observation in sputtered and evaporated thin films that the grain size is of the same order as the film thickness. The grains of the Co layer, however, are approximately $11 \times 20 \mathrm{~nm}$ and are very similar for Co_25 and Co_50. A similar size of grains $d \sim$ $11.5 \mathrm{~nm}$ is also estimated from the width of the Co peak from $\mathrm{x}$-ray diffraction measurements. Therefore there is no visible microstructural difference in the Co layers.

An increased coercivity in exchange coupled systems is a clear indicator for a dominance of domain wall pinning, as the AF domain walls act as pinning sites for the neighboring FM domains. ${ }^{22}$ Thus if we presume the grains to evolve (decrease) with increasing number of layers in a ML stack then an increase in the number of AF domain walls or increased grain boundaries is expected for those domain walls to form. In the case, that the evolution is interrupted (as in the present case), the number of AF domains will remain similar. In any case, this would concomitantly influence the FM domains.

When comparing the ML microstructures, particularly for Co_25 and Co_50, the enhanced coercivities of the FM layers do not appear to correlate in a systematic way with the AF grains. Due to a distribution of grain size, one can expect exchange decoupled AF grains (associated with individual grain spins) at the interface and exchange coupled FM grains. The FM and AF layer coupling can be via exchange and dipole-dipole interactions. However, the additional anisotropy giving rise to the enhanced coercivity can also have its origin within the bulk of the $\mathrm{AF}$ layer due to the grain structure that affects the AF magnetocrystalline anisotropy. ${ }^{29}$ Hence the enhanced coercivity might be a combination of the effects in both the bulk and interfacial grain spins of the AF layers.

Since the XTEM pictures do not show a significant variation of the grain structures with an increase in the spacer layer thickness, the coupling of the interfacial grains can be considered to be responsible for the increase of the coercivity.
It may be possible that due to the different oxidation states of the AF layer, i.e., $\mathrm{CoO}, \mathrm{Co}_{3} \mathrm{O}_{4}$, and $\mathrm{Co}_{2} \mathrm{O}_{3}$, the individual grains are coupled differently to the FM grains. Such different oxidation states may originate from changes in the deposition conditions within the chamber while depositing a thicker spacer layer. $\mathrm{CoO}$ in a stoichiometric relationship $\mathrm{Co}: \mathrm{O}=1$ : 1 is not the only binary oxide phase that forms under readily attainable oxygen partial pressures. The thermodynamically favored form of the cobalt oxide is often $\mathrm{Co}_{3} \mathrm{O}_{4}$. In contrast to the two cobalt oxides mentioned above, the metastable form $\mathrm{Co}_{2} \mathrm{O}_{3}$ may be difficult to form.

\section{Specular and off-specular neutron scattering}

\section{Scattering geometry}

The neutron scattering geometry is shown in Fig. 8. We define the ML surface in the $x-y$ plane and the $z$ axis along the surface normal. In the specular scattering geometry

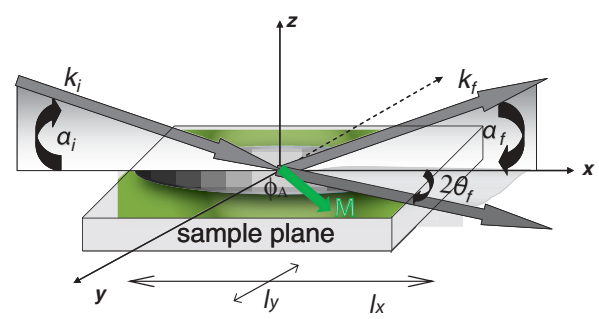

FIG. 8. (Color online) Schematic of the neutron scattering geometry. In reflection geometry, the beam is collimated in the reflection plane and relaxed along the $y$ axis, whereas in the GISANS geometry scattering along the $y$ axis is resolved. Here, $\vec{k}_{i}$ is the incident wave vector at an angle $\alpha_{i}$. The scattered wave vector $\overrightarrow{k_{f}}$ makes an angle $\alpha_{f}$ and $2 \theta_{f}$ along two different scattering planes. The grey shaded region represents the coherence ellipse covering several (or single) domains (shaded in green) and the mean magnetization making an angle $\phi_{A}$ with the polarization axis, which is along the $y$ axis. 


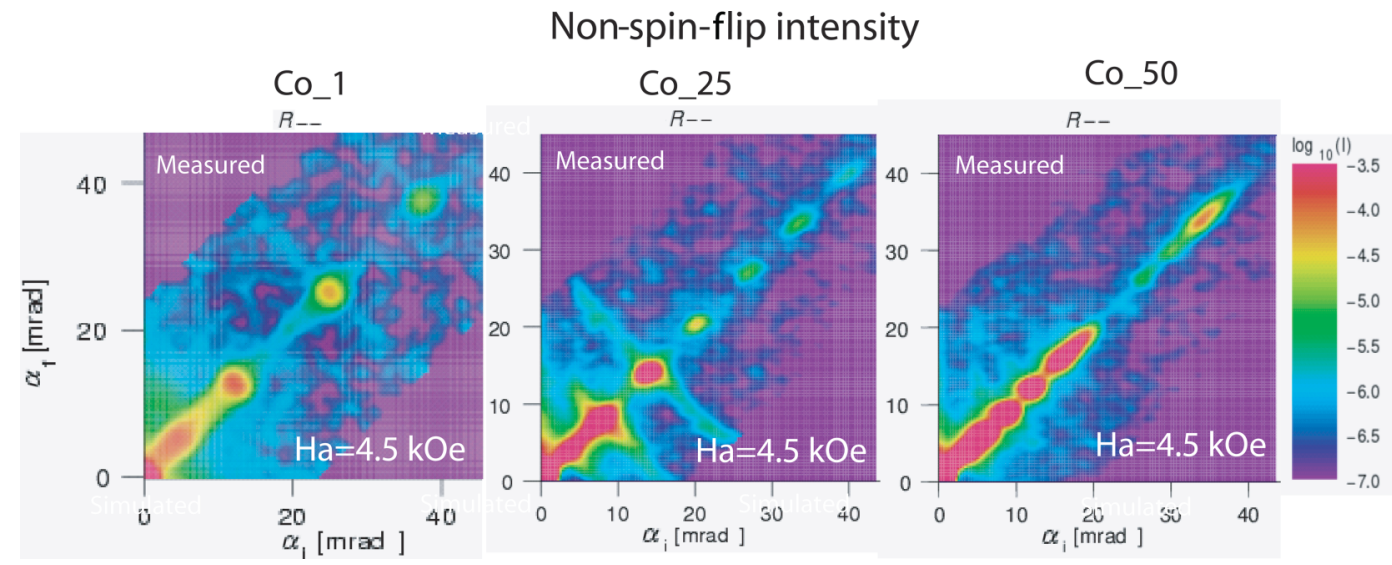

FIG. 9. (Color online) NSF intensity maps $\left(R_{--}\right)$from $\mathrm{Co} / \mathrm{CoO} / \mathrm{Au}$ MLs measured on HADAS/TREFF at saturating field along the decreasing branch of the hysteresis loops for Co_1 and Co_25 and Co_50 ML samples after field cooling at $4.5 \mathrm{kOe}$ and measured at $10 \mathrm{~K}$. The color bar encodes the scattered intensity on a logarithmic scale.

(i.e., angle of incidence $\alpha_{i}$ equal to the exit angle $\alpha_{f}$ ), the reflectivities follow from energy and in-plane momentum conservation laws as normal wave-vector transfers $\vec{Q}_{\perp}$ are probed. However, when the in-plane translational symmetry is broken by interface waviness (roughness) or by magnetic domains on a length scale shorter than the in-plane projection of the neutron coherence length $l_{\|}$along $\vec{Q}_{\|}\left(=\vec{Q}_{x}, \vec{Q}_{y}\right)$ then the off-specular scattering contributions along the in-plane momentum transfer vector $\left(\vec{Q}_{\|}\right)$arise.

At grazing incidence, there can be three scattering geometries: specular reflection, scattering in the plane of incidence (off-specular scattering), and scattering perpendicular to the plane of incidence (Grazing Incidence SANS). We can estimate the extent of correlation lengths from the three equations of momentum transfers along the three different axis owing to the scattering geometry for small angles:

$$
\begin{aligned}
\vec{Q}_{z}=\vec{Q}_{\perp} & =\frac{2 \pi}{\lambda}\left[\sin \left(\alpha_{\mathrm{i}}\right)+\sin \left(\alpha_{\mathrm{f}}\right)\right] \simeq \frac{2 \pi}{\lambda}\left(\alpha_{i}+\alpha_{f}\right), \\
\vec{Q}_{x} & =\vec{Q}_{\|}=\frac{2 \pi}{\lambda}\left[\cos \left(\alpha_{f}\right) \cos \left(2 \theta_{f}\right)-\cos \left(\alpha_{i}\right)\right] \\
& \simeq \frac{2 \pi}{\lambda}\left(\frac{\alpha_{i}^{2}}{2}-\frac{\alpha_{f}^{2}}{2}-2 \theta_{f}^{2}\right), \\
\vec{Q}_{y} & =\vec{Q}_{\|}^{\prime}=\frac{2 \pi}{\lambda} \cos \left(\alpha_{f}\right) \sin \left(2 \theta_{f}\right) \simeq \frac{4 \pi}{\lambda}\left(\theta_{f}\right) .
\end{aligned}
$$

Here, the incident wave-vector defined by $\vec{k}_{i}$, makes an angle $\alpha_{i}$ in the $x-z$ plane with respect to the $x$ axis, while the scattered wave vector $\overrightarrow{k_{f}}$ makes angle $\alpha_{f}$ in the $x-z$ plane and also $2 \theta_{\mathrm{f}}$ in the $x-y$ plane (relevant for diffuse scattering). Different length scales $\xi=\frac{2 \pi}{\bar{Q}}$ ranging from nanometers to micrometers can be accessed by using different scattering geometries in most practical cases. Specular scattering provides the scattering potential of the ML perpendicular to the film plane. The typical probed length scales are in the range $3 \mathrm{~nm}<\zeta<1 \mu \mathrm{m}$. Off-specular scattering scans provide the lateral correlations along $\vec{Q}_{x}(500 \mathrm{~nm}<\xi<50 \mu \mathrm{m})$, whereas grazing incidence SANS scans probe the surface $(3 \mathrm{~nm}<\xi<100 \mathrm{~nm})$ along $\vec{Q}_{y}$. From the above equations, one may also note that for a given geometry when $\alpha_{i} \sim \alpha_{f} \sim \theta_{f} \ll 1$, the projection $\vec{Q}_{y} \sim$ $\vec{Q}_{z} \gg \vec{Q}_{x}$.

\section{NSF scattering}

The scattering-length densities (SLD) of a magnetic specimen are given by either the sum or difference of the nuclear $\left(\rho_{n}\right)$ and magnetic $\left(\rho_{m}\right)$ components. The \pm signs refer to the spin-up and spin-down states of the incident neutron beam with respect to the magnetization of the sample. The non-spin-flip (NSF) scattering amplitude provides information about $\rho_{n} \pm \rho_{m} \cos \phi_{\mathrm{A}}$, and the spin-flip (SF) channels measure $\rho_{\mathrm{m}}^{2} \sin ^{2} \phi_{\mathrm{A}}$, if the domain size is larger than the projection of the neutron coherence length along the sample plane $\left(l_{\|}\right)$. Here, $\phi_{\mathrm{A}}$ is the angle between the magnetization $M$ and the applied field $H_{a}$, which corresponds usually to the neutron quantization axis.

a. Intensity maps. Next, we show the specular and offspecular NSF intensity maps in Fig. 9 for the Co_1, Co_25, and Co_50 samples corresponding to the channel $R_{--}$. The intensity along the diagonal $\alpha_{i}=\alpha_{f}$ is the specular reflection along the scattering vector $Q_{\perp}$. In the experimental geometry, only $\vec{Q}_{x}$ is resolved whereas the signal along $\vec{Q}_{y}$ is integrated because the collimation along the $y$ axis is relaxed. The NSF intensities are shown at a saturating field along the decreasing branch of the respective hysteresis loops where the MLs are in the single domain state. The observed superlattice peaks from the specimens (see Fig. 9) confirm the periodicity of the multilayer structure. The off-specular scattering along the Bragg sheets occurs due to pronounced structural vertical correlation of each of the MLs.

$b$. Specular scattering. The neutron reflectivity does not only carry information on the mean magnetization direction but also on the layer-by-layer vectorial magnetization. In corroboration to the drop of the net magnetization at the first switching field ( $\left.H_{a}=0.75 \mathrm{kOe}\right)$ along the hysteresis loop of the Co_1 ML, the fits to the neutron reflectivity data, indeed, show the switching of one out of the twenty FM layers at an applied field $H \simeq 1.0 \mathrm{kOe}$. Similar to the Co_1 ML, ${ }^{32}$ we find layer-by-layer flipping for the Co_25 and C0_50 MLs as well, 


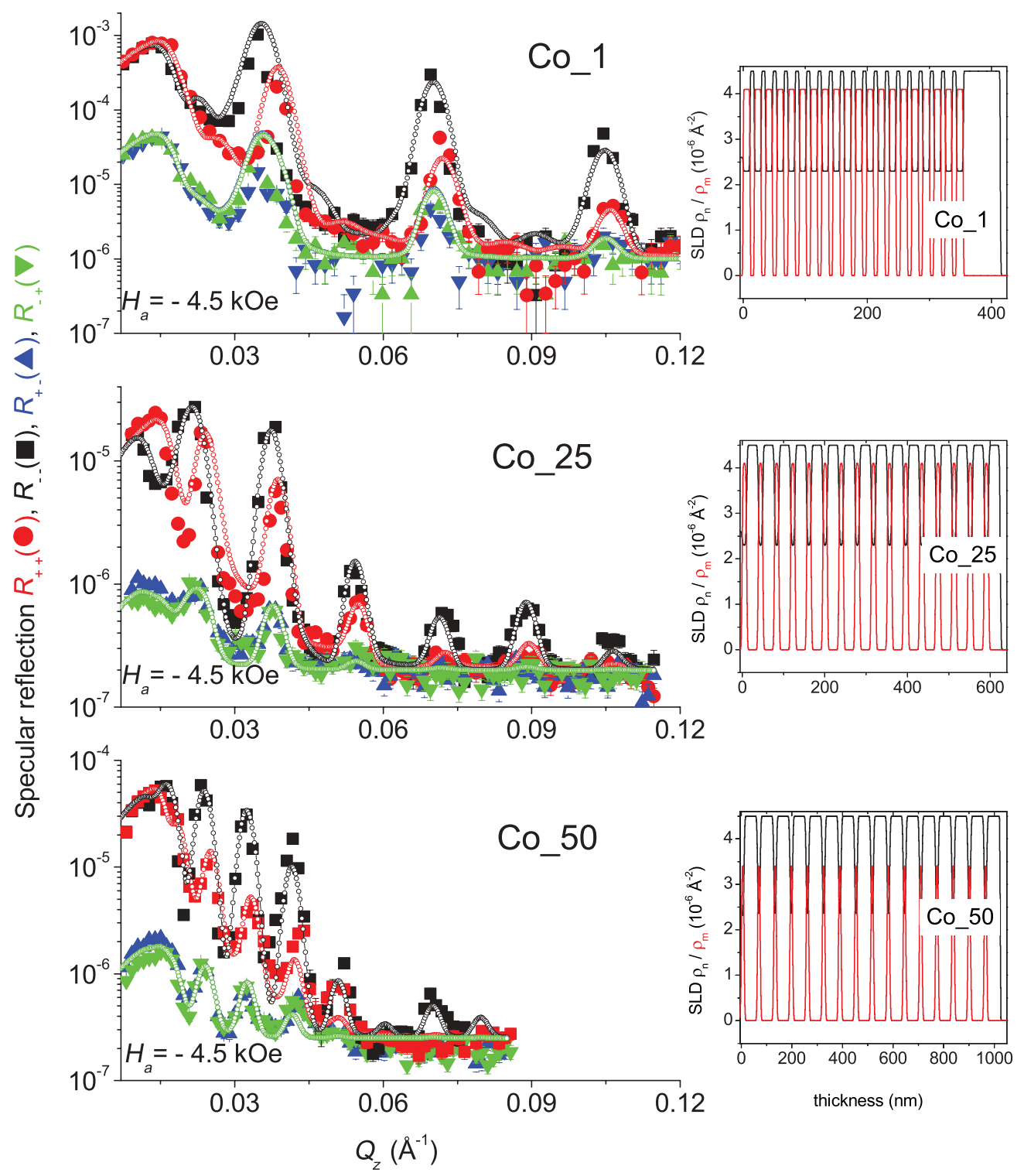

FIG. 10. (Color online) Specular reflectivity patterns (solid symbols) along with their best fits (open symbols) for the NSF $\left[R_{++}\right.$(red) and $R_{--}$(black)] and SF $\left[R_{-+}\right.$(green) and $R_{+-}$(blue)] channels measured at a saturation field, for the MLs with different spacer layer thicknesses. $\vec{Q}_{z}=\frac{2 \pi}{\lambda}\left[\sin \left(\alpha_{i}\right)+\sin \left(\alpha_{f}\right)\right]$, where $\alpha_{i}$ and $\alpha_{f}$ are the incident and exit angles, respectively. The fits shown here are done by considering model A for the MLs Co_1 and Co_25 and model C for the ML Co_50. The corresponding nuclear (black) and magnetic (red) SLDs are shown alongside.

which is indicated by their multiple switching fields along the respective hysteresis loops.

Figure 10 shows the specular reflectivity data (NSF and SF) corresponding to the three MLs at a saturation field $\left(H_{a}=-4.0 \mathrm{kOe}\right)$ on a logarithmic scale. The relative variation of the multilayer Bragg peak intensities due to different periodicities of the MLs is quite evident here. Earlier, the layer magnetizations for Co_25, measured at their first switching field by Paul et al. ${ }^{20}$ revealed that at least four layers from the stack have flipped and the remaining twelve layers are at the onset of flipping. Here, we find the layer magnetizations for the Co_50 ML, also remain collinear at its first switching field, whereby nine of the sixteen layers have flipped with the field. The value of the mean magnetization angle $\phi_{A}$ for the individual layers in the stack $\left(0^{\circ}\right.$ or $180^{\circ}$ with respect to the field) are taken from the fitted values of the specular patterns (NSF and SF). We do not find any significant increase in the SF specular signals confirming their nonuniform reversal that is expected for these MLs, as we measure along the decreasing branch of the first field cycle. The best fits to the reflectivity data revealed a good agreement with the nominal thicknesses and the $\rho_{m}$ and $\rho_{n}$ values as listed in Table II. The other parameters such as interface roughness are kept similar for all samples. The respective nuclear and magnetic SLD values are plotted alongside.

Note that the bulk value of the Co moment is $\sim 1.73 \mu_{B} /$ atom, here $\mu_{B}$ designates the Bohr magneton. ${ }^{35}$ The estimated magnetic moment from the corresponding values of $\rho_{m}$ as obtained from the least square fit to the Co_1 ML neutron reflectivity profile measured at saturation is $\sim 1.66 \mu_{B}$ /atom 
TABLE II. Fit parameters extracted from the PNR results. $\rho_{n}$ and $\rho_{m}$ designate the nuclear and magnetic scattering length densities, respectively. In sample $\mathrm{Co}_{-} 1$, there are no spacer layers between the $\mathrm{Co} / \mathrm{CoO}$ bilayers. Also given are the respective magnetic moments as calculated from the magnetic scattering length densities and the exchange energy E. The magnetic moments are calculated following model A: considering no dead layer, model B: considering $1.0 \mathrm{~nm}$ of dead layer, and model C: considering reduced moment for the entire magnetic layer. The Au layer in Co_1 protects the sample against oxidation.

\begin{tabular}{|c|c|c|c|c|c|c|}
\hline Multilayer & & $\mathrm{Au}$ & $\mathrm{CoO}$ & $\mathrm{Co}$ & Co-Au (dead layer) & error \\
\hline Co_1 & thickness (nm) & 52.6 & 7.1 & 11.0 & - & \pm 0.2 \\
\hline \multirow[t]{2}{*}{ (A) } & $\rho_{n}\left(\times 10^{-6} \AA^{-2}\right)$ & 4.5 & 4.5 & 2.3 & - & \pm 0.2 \\
\hline & $\rho_{m}\left(\times 10^{-6} \AA^{-2}\right)$ & 0.0 & 0.0 & 4.1 & - & \pm 0.1 \\
\hline Co_1 & thickness (nm) & 52.6 & 7.1 & 10.0 & 1.0 & \pm 0.2 \\
\hline \multirow[t]{4}{*}{ (B) } & $\rho_{n}\left(\times 10^{-6} \AA^{-2}\right)$ & 4.5 & 4.5 & 2.3 & 2.3 & \pm 0.2 \\
\hline & $\rho_{m}\left(\times 10^{-6} \AA^{-2}\right)$ & 0.0 & 0.0 & 4.1 & 0.0 & \pm 0.1 \\
\hline & magnetic moment $\left(\mu_{B} /\right.$ atom $)$ & & & 1.66 & & \pm 0.1 \\
\hline & $\mathrm{E}\left(\mathrm{erg} \mathrm{cm}^{-2}\right)$ & & & 0.62 & & \pm 0.1 \\
\hline Co_25 & thickness (nm) & 22.5 & 5.5 & 11.0 & & \pm 0.2 \\
\hline \multirow[t]{4}{*}{$(\mathrm{A})^{-}$} & $\rho_{n}\left(\times 10^{-6} \AA^{-2}\right)$ & 4.5 & 4.5 & 2.3 & & \pm 0.2 \\
\hline & $\rho_{m}\left(\times 10^{-6} \AA^{-2}\right)$ & 0.0 & 0.0 & 4.1 & & \pm 0.1 \\
\hline & magnetic moment ( $\mu_{B} /$ atom $)$ & & & 1.66 & & \pm 0.1 \\
\hline & $\mathrm{E}\left(\mathrm{erg} \mathrm{cm}^{-2}\right)$ & & & 0.92 & & \pm 0.1 \\
\hline Co_50 & thickness (nm) & 48.0 & 5.0 & 11.0 & & \pm 0.2 \\
\hline \multirow[t]{2}{*}{ (A) } & $\rho_{n}\left(\times 10^{-6} \AA^{-2}\right)$ & 4.5 & 4.5 & 2.3 & & \pm 0.2 \\
\hline & $\rho_{m}\left(\times 10^{-6} \AA^{-2}\right)$ & 0.0 & 0.0 & 4.1 & & \pm 0.1 \\
\hline Co_50 & thickness (nm) & 48.0 & 5.0 & 10.0 & 1.0 & \pm 0.2 \\
\hline \multirow[t]{2}{*}{ (B) } & $\rho_{n}\left(\times 10^{-6} \AA^{-2}\right)$ & 4.5 & 4.5 & 2.3 & 2.3 & \pm 0.2 \\
\hline & $\rho_{m}\left(\times 10^{-6} \AA^{-2}\right)$ & 0.0 & 0.0 & 4.1 & 0.0 & \pm 0.1 \\
\hline Co_50 & thickness (nm) & 48.0 & 5.0 & 11.0 & & \pm 0.2 \\
\hline \multirow[t]{4}{*}{ (C) } & $\rho_{n}\left(\times 10^{-6} \AA^{-2}\right)$ & 4.5 & 4.5 & 2.3 & & \pm 0.2 \\
\hline & $\rho_{m}\left(\times 10^{-6} \AA^{-2}\right)$ & 0.0 & 0.0 & 3.5 & $\rightarrow$ reduced & \pm 0.1 \\
\hline & magnetic moment ( $\mu_{B} /$ atom $)$ & & & 1.45 & & \pm 0.1 \\
\hline & $\mathrm{E}\left(\mathrm{erg} \mathrm{cm}^{-2}\right)$ & & & 1.35 & & \pm 0.1 \\
\hline
\end{tabular}

\pm 0.05 . Note that this is $17.4 \%$ less when compared with the moment obtained from the magnetometric measurements using the SQUID/PPMS (see Table I). One may recall that $\rho_{m}=M_{\mathrm{FM}} 2.853 \times 10^{-9} \AA^{-2} \mathrm{~cm}^{3} \mathrm{emu}^{-1}$. The magnetic moment for the Co_50 ML as obtained from the PNR data fits, is $\sim 1.45 \mu_{B}$ /atom \pm 0.05 (13\% reduction from the Co_1 value). The reduced magnetic moment of the Co layers as obtained from the magnetometry measurements lead us to infer that there can be plausible magnetic dead layers at the Co-Au interfaces as we increase the Au spacer thickness. Such formation of dead layers on magnetron sputtered samples are commonly attributed to the interdiffusion that occurs during the deposition process. ${ }^{36}$

c. Models for fitting. In order to verify the formation of weakly coupled noncollinear domains at the interface, we compare the PNR profiles for the Co_1 and Co_50 specimens. These systems were chosen for comparison because the changes of the magnetic moment are maximum for these two MLs.

First, we compare the NSF simulated data (on a linear scale) over a certain range of $\vec{Q}_{z}$ where the changes are explicit, considering different probable models. The simulations in Fig. 11 are shown for both the MLs as we consider three models with (A) no magnetic dead layer (closed symbols), (B) $1.0 \mathrm{~nm}$ of magnetic dead layer (open symbols) at the Co-Au interfaces (see Table II), and a third model (C) with reduced moment throughout the entire Co layer thickness for the Co_50 ML (lines). The Co_1 ML obviously does not have Au spacers after each $\mathrm{Co}-\mathrm{CoO}$ bilayer rendering model (C) irrelevant for it. One can clearly distinguish the impact of the models on the profiles and therefore our inferences from the fits can be considered unambiguous.

d. Spin asymmetry. Furthermore, the measured spinasymmetry (SA) profile is plotted versus $\vec{Q}_{z}$ in Fig. 12. The spin asymmetry is expressed as the ratio of the difference and sum of $R_{++}$and $R_{--}$reflectivities measured at a saturation field of $-4.5 \mathrm{kOe}$. This normalized difference is sensitive to the magnetization profile across the film and is less sensitive to interface roughness.

We follow the fit qualities in Figs. 11 and 12 for the Co_1 ML and Co_50 ML profiles using the model A, B, and C. Note the different ranges of the $\vec{Q}_{z}$ in Fig. 11 chosen for the two samples in order to compare the differences of model fits. One can see from both figures that the fit quality deteriorates for the case with model B (dead layer) in case of Co_1 ML. This confirms that there are no dead layers in this specimen. All Co layers (in each bilayer repetition) have an uniform magnetization throughout the entire thickness of the layer. A very similar situation is encountered for the Co_25 ML as well. However, from the Co_50 ML profile, one can see that a slight improvement in the fit quality has been achieved by using model $\mathrm{C}$, i.e., by considering a $13 \%$ reduction in the moment for the entire Co layer $(11.0 \mathrm{~nm})$. No significant improvement in the fit quality can be achieved by using model B (dead layer at the Co-Au interface). 

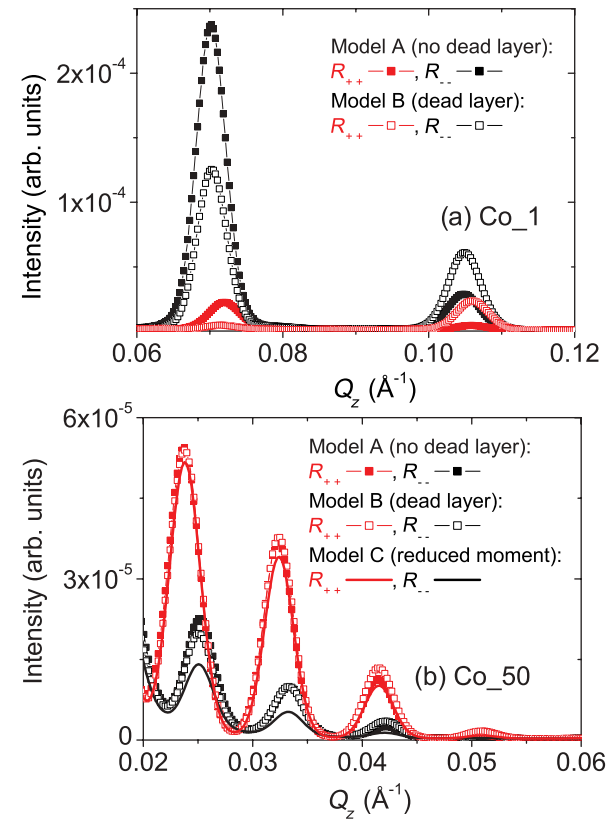

FIG. 11. (Color online) NSF simulations $\left[R_{++}\right.$(red) and $R_{--}$ (black)] with $\vec{Q}_{z}$ for the (a)Co_1 and (b) Co_50 MLs. The simulations are shown to compare for the models considering (A) no magnetic dead layer (closed symbols), (B) $1.0 \mathrm{~nm}$ of magnetic dead layer (open symbols) at the Co-Au interface, and a third model (C) with reduced magnetic moment throughout the entire Co layers for the Co_50 ML (lines). Note the different ranges of $\vec{Q}_{z}$ chosen for the two samples in order to show the differences in model fits distinctly.

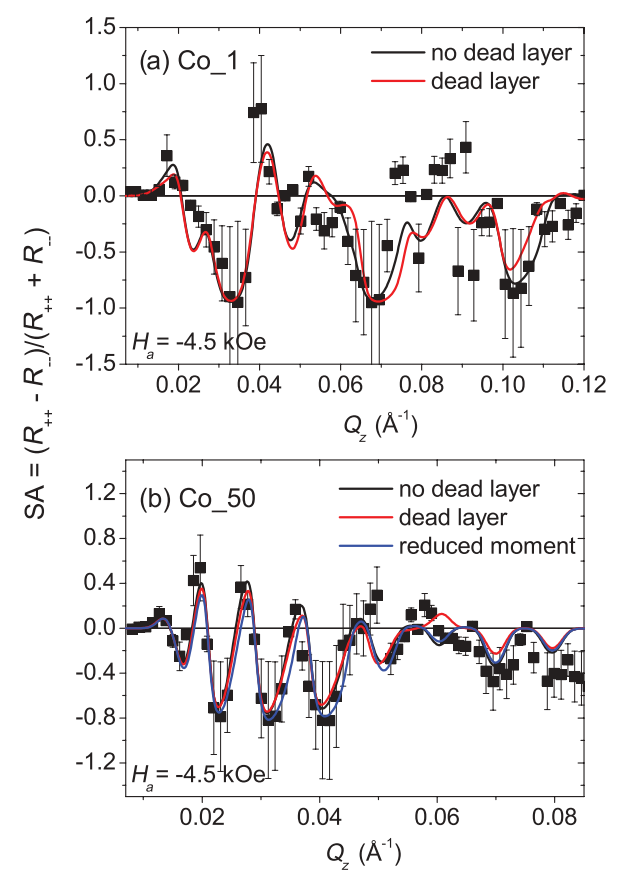

FIG. 12. (Color online) Spin asymmetry (SA) (black square) with $\vec{Q}_{z}$ in order to compare the magnetization in the (a) Co_1 and (b) Co_50 MLs. The simulations are shown for the models considering (A) no magnetic dead layer (black line), (B) $1.0 \mathrm{~nm}$ of magnetic dead layer (red line) at the Co-Au interface, and a third model (C) with reduced magnetic moment throughout the entire Co layers for the Co_50 ML (blue line).

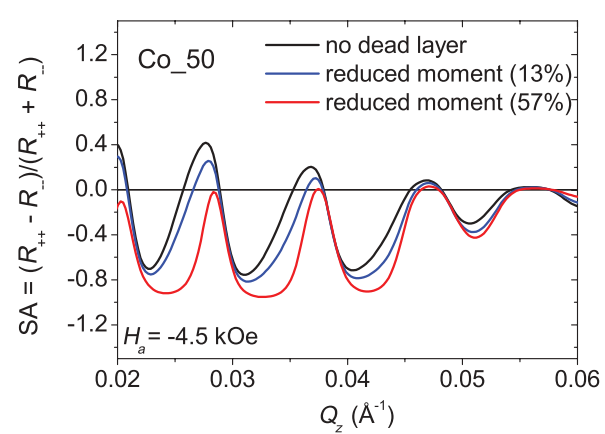

FIG. 13. (Color online) Simulated SA is plotted with $\vec{Q}_{z}$ for the Co_50 ML considering different degrees of reduction in magnetization of the Co layer as obtained from the PNR data and also from the PPMS data.

Figure 13 shows the simulated SA versus $\vec{Q}_{z}$ for various reductions of the magnetic moment of the Co layer in Co_50 ML. One can see that when the moment (or $\rho_{m}$ ) is reduced by $57 \%$ (which is estimated from the PPMS measurements) a strong deviation is encountered as compared to the best fit which is simulated considering only a $13 \%$ reduction in the magnetic moment. Note that these values are compared for the apparent saturation field measurements, thus one can rule out the possibility of canting in the film plane (however out-of-plane canting may be possible).

e. Discrepancies in magnetic moment. In the present case, from the changes in $M_{\mathrm{FM}}$ as obtained from PPMS (see Table I) and as obtained from PNR (see Table II), the exchange coupling $E$ can be calculated. It turns out that $E \sim 0.75 \pm 0.05 \mathrm{erg} \mathrm{cm}^{-2}$ is almost independent of the spacer layer thickness of the MLs as obtained from the PPMS measurements. The $E$ values, as obtained from the PNR measurements however, show a two times increase for the Co_50 $\left(\sim 1.35 \pm 0.1 \mathrm{erg} \mathrm{cm}^{-2}\right) \mathrm{ML}$ as compared to the Co_1 ML. This of course follows from the respective difference in reduced magnetizations (particularly for the Co_50 ML) as obtained from the two techniques used.

Discrepancies in the estimates of the magnetic moment are commonly reported for SQUID based magnetometers and PNR measurements. ${ }^{37}$ This becomes more visible, probably for oxidized layers, due to plausible inhomogeneities. Measurements at TREFF were done with a $2.0 \mathrm{~mm}$ beam diverging by $\sim 0.1^{\circ}$ at a distance of $1500 \mathrm{~mm}$ from the $15-\mathrm{mm}$ sample along $\vec{Q}_{x}$. The neutron coherence lengths $l_{x}$ (along $\vec{Q}_{x}$ ) and $l_{y}$ (along $\left.\vec{Q}_{y}\right)^{38}$ thereby turn out to be few micrometers and few angstrom, respectively, which can be estimated using the uncertainties in $\vec{Q}_{x}$ and $\vec{Q}_{y}$ as

$$
\begin{aligned}
l_{x} \sim \frac{1}{\Delta Q_{x}} & \sim \frac{1}{\frac{\pi}{\lambda} \sqrt{\left(\alpha_{i} \Delta \alpha_{i}\right)^{2}+\left(\alpha_{f} \Delta \alpha_{f}\right)^{2}}}, \\
l_{y} & \sim \frac{1}{\Delta Q_{y}} \sim \frac{1}{\frac{2 \pi}{\lambda} \Delta \theta_{f}} .
\end{aligned}
$$

Here, $l_{x}$ being $\ll$ than the illuminated sample area $(\sim 2.0$ $0.65 \mathrm{~mm}$ ), the intensities on the detector are an incoherent sum of the coherently scattered intensities from the coherent ellipse. This can make a significant difference for samples with laterally and vertically inhomogeneous magnetic entities 

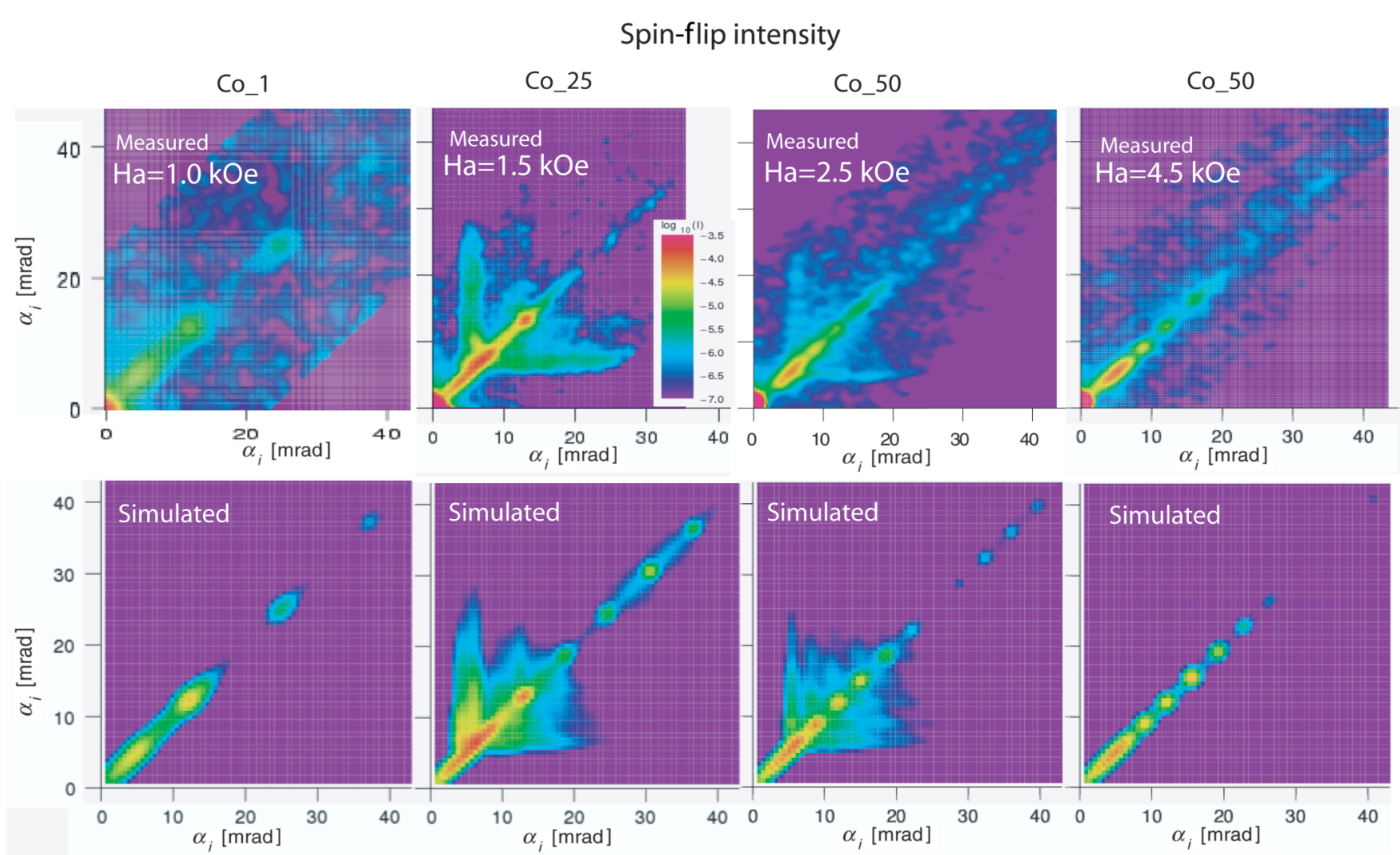

FIG. 14. (Color online) SF intensity maps $\left(R_{+-}\right)$along with their simulations within DWBA from Co/CoO/Au MLs measured on HADAS/TREFF at the first switching fields along the decreasing branch of the hysteresis loops for Co_1 and Co_25 and Co_50 ML samples after field cooling at $4.0 \mathrm{kOe}$ and measured at $10 \mathrm{~K}$. The color bar encodes the scattered intensity on a logarithmic scale.

varying from one coherence volume to the other. The PPMS measurements, on the other hand, are from a signal averaged over $5 t_{\mathrm{FM}}-\mathrm{mm}^{3}$ sample volume.

\section{SF scattering}

a. Intensity maps. Figure 14 shows intensity maps for the Co_1, Co_25, and Co_50 samples corresponding to the channel $R_{+-}$. The SF intensities are shown at a field that is close to the first switching fields along the decreasing branch of the respective hysteresis loops. These intensities eventually disappear at saturation, demonstrating their magnetic origin. A small contribution from the NSF intensities appears in the SF channels due to a reduction of the efficiency of $\approx 5 \%$ of the polarizer and the analyzer components. Note that no Bragg sheets are visible in the SF channels unlike that in the NSF channels.

Here, we consider three possible scenarios for the Co_1 ML for fields close to the coercive field: (i) Paul et al. ${ }^{32}$ have shown earlier that the reflectivity profile near coercivity is best simulated considering an almost equal number of layers oriented along the applied field direction and opposite to it. (ii) The magnetization is close to zero due to the formation of a multidomain state with random orientation of the domains, and (iii) the magnetization is oriented along an axis perpendicular to the polarization axis, corresponding to a coherent rotation of magnetization. In all these three cases, the projection of the longitudinal magnetization onto the neutron polarization axis ( $y$ axis) is proportional to $\left\langle\cos \phi_{A}\right\rangle(=0)$, while the projection of the transverse component with respect to the polarization axis onto the $x$ axis is proportional to $\left\langle\sin ^{2} \phi_{A}\right\rangle$. However, in the case of a random distribution of domain magnetization directions, the dispersion is $\left\langle\cos ^{2} \phi_{A}\right\rangle-\left\langle\cos \phi_{A}\right\rangle^{2} \neq 0$. For a coherent rotation this dispersion is essentially zero. Thus one can distinguish between a situation of random distribution of domains and that between a coherent rotation. In case of domains that are smaller than the neutron coherence length along the $x$ axis, off-specular scattering is expected as well. The situation becomes more involved when an equal number of layer magnetizations is oriented along and opposite- - but are strictly collinear - to the polarization axis. It is then difficult (or even impossible) to infer the domain size as there is no SF off-specular scattering in absence of fluctuations around the mean magnetization $\mathbf{M}$ direction even if the domains are smaller than the neutron coherence length.

The absence of well defined Bragg sheets in Fig. 14, in the off-specular scattering from Co_25 and Co_50 MLs indicates a lack of vertical correlations. In contrast to the Co_1 ML, both Co_25 and Co_50 MLs show a significant increase in the off-specular intensities. They occur due to fluctuations of the magnetization of the domains around the mean magnetization angle indicating an instability that is induced in the system at the onset of flipping of the magnetization of the layers. Flipping is likely when the size of the domains becomes comparable to the width of the domain walls.

b. DWBA simulations. The specular and the off-specular intensity is simulated within the distorted wave Born 
approximation (DWBA). ${ }^{38}$ The simulations are conducted by taking into account spin-dependent reflection and refraction. Finally, the cross section is convoluted with the instrumental resolution function (see Fig. 14). Inhomogeneities of the ML like magnetic roughness at the interfaces are taken into account to first order starting from an ideal multilayer with flat interfaces. We assume for all measurements that the mean magnetization is collinear with the neutron polarization axis, which is along the $y$ axis. Note that the coherence area is substantially extended along the $x$ axis (see Fig. 8). This area is restricted via the uncertainty in the momentum transfers ( $\Delta \mathrm{Q}_{x, y} \sim \frac{2 \pi}{l_{x, y}}$ ) along the $x$ and $y$ directions. The uncertainties are a consequence of the angular divergences due to the beam collimation opted in the measurements.

The off-specular scattering gradually disappears when the field becomes larger than the first switching field (see Fig. 14). Within our model, we allow $\mathbf{M}$ to fluctuate from domain to domain around the mean angle by $\Delta \phi_{A}=30^{\circ}$ averaged over the coherence volume for Co_25 and Co_50. These fluctuations can be longitudinal $\left\langle\cos \left(\delta \phi_{A}\right)\right\rangle(\Delta \mathbf{M} \| \mathbf{M})$ as well as transverse $\left\langle\sin ^{2}\left(\Delta \phi_{A}\right)\right\rangle(\Delta \mathbf{M} \perp \mathbf{M})$. The structural parameters are obtained from the fits to the specular patterns.

Transmission and reflection amplitudes show singularities at the points of total reflection, i.e., at the critical edges. Figure 14 clearly shows these singularities, i.e., the Yoneda wings, which in turn are accompanied by an enhancement of the diffuse scattering. Such enhancements can be seen in the SF maps in cases when the domains are smaller than the neutron coherence length along the $x$ axis, i.e., as and when the coherence ellipse covers several domains. One usually encounters an asymmetry of the scattered neutrons in the SF channels due to the inverse population of the incoming and the outgoing neutrons selected by the polarizer (different critical edges for up and down neutrons) and flipped by the spin flippers. ${ }^{38}$ One can also see, particularly for the Co_50 data and its simulation, that the Yoneda wings are associated with streaks running parallel to the $\alpha_{i}$ and $\alpha_{f}$ axes. These streaks are commonly observed when the SLD values of one of the constituents of the ML form a shallow potential well (Co) with respect to a wider and higher SLD value $(\mathrm{Au})$. The effect is related to the difference in the phases of the transmitted and reflected waves.

It is well known that a decreasing domain size leads to a concomitant increase in the number of grain boundaries (as domains can be associated with the grain size) and thereby an increase in the number of uncompensated spins in the $\mathrm{AF}$ as in the case of Co_1 type (nonseparated) MLs. ${ }^{12,32}$ However, for the Co_1 ML, the magnetic correlation length cannot be properly estimated. This is because, at the reversal point, $50 \%$ of the layers are directed along the applied field and the remaining $50 \%$ are directed opposite to the applied field direction. Thus the net magnetization is close to zero. Furthermore, there are no indications of small scale variations around the mean magnetization angle close to the critical angle of total reflection (even at its reversal point) and also that these domains are either vertically uncorrelated (no Bragg sheets are observed in the SF channels) and/or larger than the neutron coherence length projected along $\vec{Q}_{x}$. Whereas, in the cases Co_25 and Co_50 ML specimens, the typical FM vertically uncorrelated domain sizes are of $\approx 1-2 \mu \mathrm{m}$ (estimated from the observed enhanced SF scattering intensities around the total reflection edges in each of the specimens), which are consistent with previous measurements on similar samples. ${ }^{39}$ Note that we could not observe any appreciable change in the domain size with the spacer layer thickness, at least not for the separated MLs.

Generally, as the grain sizes become small enough that they are comparable to the domain wall width, where domain walls can form within one grain, the magnetization direction corresponds to the anisotropy direction varying from grain to grain. For grain sizes below the critical size, one can opt for the random-anisotropy model, which takes into account the magnetic alignment between the grains that competes with the anisotropies of the individual grains. The spontaneous spatial magnetic correlations, extended over many individual grains, thus depend strongly on grain size. ${ }^{40,41}$ Interestingly, nonevolving domain sizes in our separated MLs are in corroboration with the underlying grains (which are only of few $\mathrm{nm}$ in size) as they are also of very similar dimensions irrespective of the separation between the magnetic layers. This actually, in a way, confirms that the grain structure variation that was evident for the continuous multilayer was restricted in case of the separated multilayers. This information is significant enough as a variation in the domain sizes would have had an effect on the exchange bias as well.

\section{X-ray absorption spectroscopy \\ 1. K-edge spectroscopy}

Figure 15(a) shows a comparison of the measured Co $K$ edge XANES spectra from the MLs (solid symbols) and the reference spectra from each of the possible constituents that can produce the absorption edge for example, $\mathrm{CoO}, \mathrm{Co}_{3} \mathrm{O}_{4}$, and $\mathrm{Co}$ metal. By considering $\mathrm{CoO}, \mathrm{Co}_{3} \mathrm{O}_{4}$, and $\mathrm{Co}$ metal as the parent components, the XANES spectra of the three Co MLs are fitted (lines) with a superposition of XANES profiles of the parent components using the linear combination analysis (LCA) method. The fitting was performed using the package ATHENA $^{42}$ with the LCA tool. The fits are shown in Fig. 15(a) together with the measured XANES spectra. In this way, we estimate the weighted proportions of $\mathrm{Co}_{x} \mathrm{O}_{y}$ and Co layers.

Further, we calculate the $\mathrm{Co}$ metal, $\mathrm{Co}_{3} \mathrm{O}_{4}$ and $\mathrm{CoO}$ spectra (open symbols) using the FEFF 8.2 code, which is based on $a b$ initio multiple scattering calculations. ${ }^{43}$ The calculated spectra are shown along with the measured spectra for the MLs.

For Co metal (hexagonal), $a=2.5074 \AA$ and $c=$ $4.0699 \AA$ are used as the lattice parameters. Whereas $a=$ $4.2667 \AA$ is used for the lattice constant of $\mathrm{CoO}$ (rocksalt) structures. For $\mathrm{Co}(\mathrm{CoO})$ metal, a cluster of $40(57)$ atoms [radius of 4.5(5.0) $\AA$ ] is used to calculate the self-consistent field muffin-tin atomic potentials within the Hedin-Lundqvist exchange potential and a 80 -atom cluster with a radius of $6.0 \AA$ is used for full multiple scattering calculations. They include all possible paths within a larger cluster radius of $7.0 \AA$ (147 atoms).

Next, we vary the proportions of each of the constituents in the calculated ( $a b$ initio) spectra according to the $R$ ratio obtained from the proportional fits and compare them (open symbols) with measured XANES spectra. One can easily see 

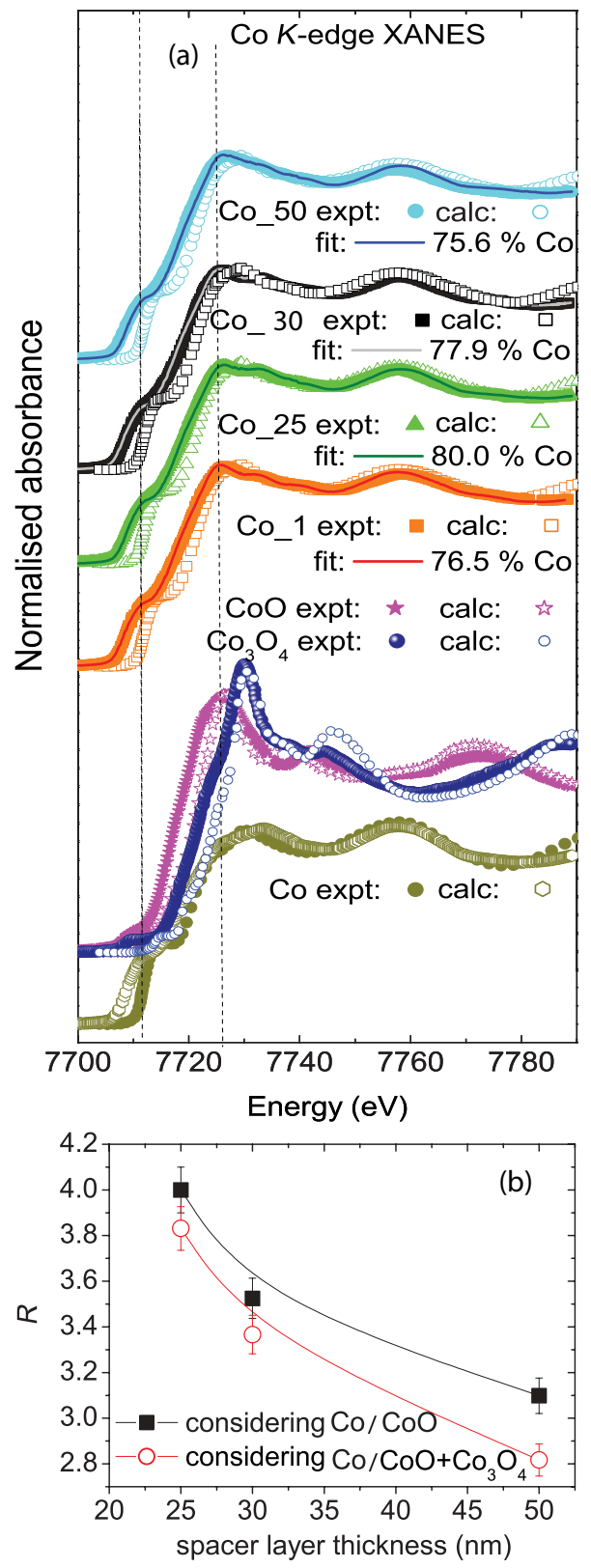

FIG. 15. (Color online) (a) Comparison of the measured Co $K$ edge normalized XANES spectra of the Co/CoO MLs (solid symbols) and their fits (lines) using the LCA method using the package ATHENA. Also included are the reference spectra for $\mathrm{CoO}, \mathrm{Co}_{3} \mathrm{O}_{4}$, and Co metal. The $a b$ initio calculated XANES spectra for the reference materials using the FEFF 8.2 code are also included. A weighted proportion of the species, with various proportions of $\mathrm{Co}$ metal, $\mathrm{Co}_{3} \mathrm{O}_{4}$, and $\mathrm{CoO}$ as obtained from the fits and are used to calculate the ML spectra, are also plotted (open circles). (b) The ratios $R$ from the $K$-edge spectra are plotted for the total thickness of the MLs using two possible scenarios discussed in the text.

that the calculated XANES spectra are in very good agreement with the corresponding features in the measured spectra of the MLs in both energy positions and shapes. This confirms the presence of multiple constituents in the MLs from $a b$ initio calculations.

The ratio of the signal, $R$ is determined by evaluating the ratio between the $\mathrm{Co}$-signal and the $\mathrm{CoO}$ - or the $\mathrm{CoO}+\mathrm{Co}_{3} \mathrm{O}_{4}$ signal as obtained from the fits. Here, we have considered two possible scenarios for the AF layer in comparing the ratios (i) with $\mathrm{CoO}+\mathrm{Co}_{3} \mathrm{O}_{4}$ content and (ii) only with $\mathrm{CoO}$ content. We have plotted these ratios in Fig. 15(b) as a function of the total ML thickness. In case of Co_1, the layer thicknesses being little different from the other MLs, the ratio cannot be strictly compared for the same thickness ratio. A better agreement with the data is obtained while considering scenario (i). The goodness-of-fit parameter ( $R$ factor) decreases by 5-30\%. This indicates that the Co MLs are composed of phase-separated regions that differ in the proportion of their respective constituents (Co metal, $\mathrm{Co}_{3} \mathrm{O}_{4}$, and $\mathrm{CoO}$ ).

From the ratio $R$ in Fig. 15(b), it is interesting to note that the XANES spectra show an increasing proportion of oxide (AF) material, which is largely compensated by a decreasing proportion of Co in the Co_25, Co_30, and Co_50 MLs. A plausible change in the deposition pressure and temperature, with increasing deposition time (while growing thicker $\mathrm{Au}$ layers), might have caused an $\approx 4 \%$ increase in the $\mathrm{Co}_{3} \mathrm{O}_{4}$ content. $\mathrm{Co}_{3} \mathrm{O}_{4}$ has an ordering temperature $\left(T_{\mathrm{N}}=40 \mathrm{~K}\right)$ lower than $\mathrm{CoO}$, which can vary depending upon the FM layer in its proximity. ${ }^{44}$ Coupling of the uncompensated AF spins within the $\mathrm{Co}_{3} \mathrm{O}_{4}$ proportion may be quite different from that within the $\mathrm{CoO}$ proportion, as they have different crystalline structures which can even lead to different anisotropy axes. Therefore the presence of multiple constituents with different magnetic ordering temperatures in a way corroborates with the magnetization loops and the multiple switching fields that has been discussed in the magnetization section.

Apart from the effect of the $\mathrm{Co}_{3} \mathrm{O}_{4}$ content, in general, an increase in the exchange bias field as has been observed here, may be associated with (a) an increase in the AF proportion (the AF thickness of our MLs is below a typical critical AF thickness of $\approx 10 \mathrm{~nm}),{ }^{45}$ (b) a decrease in the FM proportion (increasing the surface to volume effect), and (c) plausible formation of smaller AF domains ${ }^{46}$ (domains are preferably stabilized at the grain boundaries) with an increase in the total film thickness. ${ }^{12} \mathrm{~A} \approx 30 \%$ change in the AF-FM thickness ratio corroborates well with the $35 \%$ change in the exchange bias field for the corresponding MLs. The XAS data definitely provide important clues to the fact that there are indeed changes in the magnetic layer thickness or proportions that have occurred due to the spacer layer. This information is also significant enough to proceed further with the investigation.

\section{L-edge spectroscopy}

$L$-edge spectra from the $\mathrm{Co} / \mathrm{CoO}$ multilayers as measured at RT in the remanent state are shown in Fig. 16(a). As common for transition metals and transmission metal oxides, the spectra are dominated by two peaks separated by a few milli-electron-volts. The two main peaks $L_{2,3}$ arise from the spin-orbit interaction of the $2 p$ core shell. The total intensity of the peaks is proportional to the number of empty $3 d$ valence states above the Fermi level. While spectra from a metal show typically two broad peaks reflecting the width of the empty $d$ bands, oxides exhibit a multiplet structure arising from the spin and orbital momentum of the $3 d$ valence holes in the electronic ground state and from the coupled states formed 


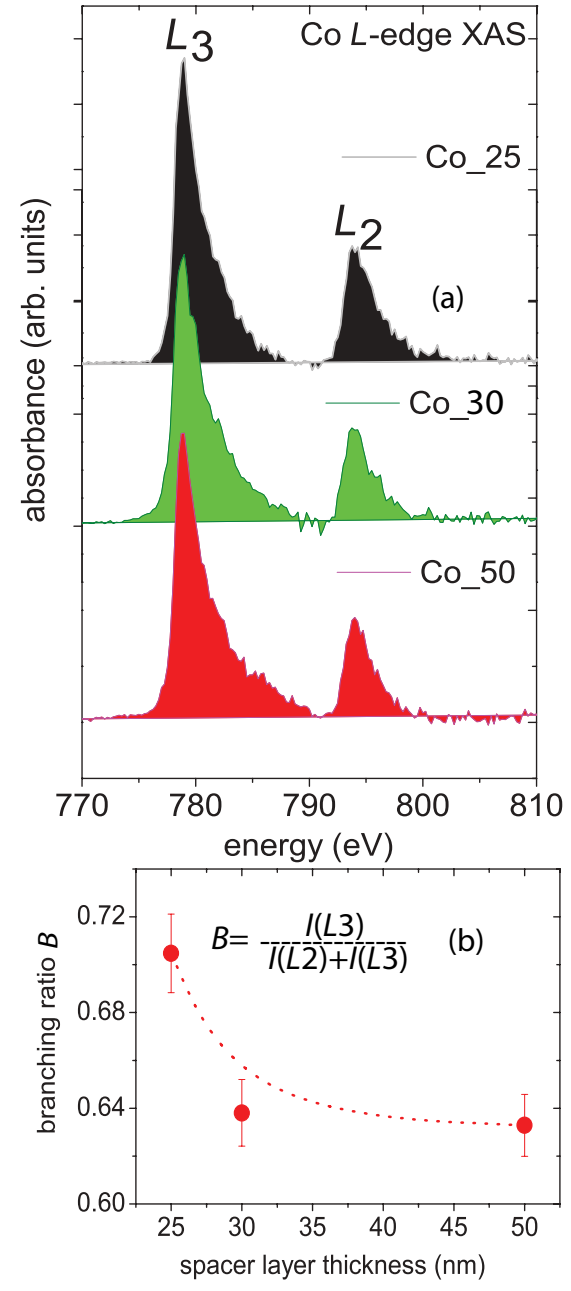

FIG. 16. (Color online) (a) Plot of the $L$-edge XAS for the multilayers. (b) Ratios of the area under the absorption peaks $L_{3}$ and $L_{2}$. The lines are guide to the eye.

after x-ray absorption between the $3 d$ valence holes and the $2 p$ core holes. $^{47}$

In our MLs we observe two broad peaks with broadened bases, a typical signature of the localized character of the $3 d$ states. We do not observe any fine structure (negating hybridization of the $d$ orbitals with the $s$ orbital of the $\mathrm{Au}$ spacer). We neither observe a shift in the absorption energies nor a change in separation of the peaks that amounts to $15.3 \mathrm{eV}$ for the MLs investigated. Therefore the amount of core-hole screening by delocalized valence electrons is negligible. ${ }^{48}$

The branching ratios $B=I\left(L_{3}\right) /\left[I\left(L_{2}\right)+I\left(L_{3}\right)\right]$ (see Ref. 49) are calculated from the area under the $L_{2}$ and $L_{3}$ peaks as shown in Fig. 16(b) using the IFEFFIT package. ${ }^{42}$ The advantage of using $B$ is the minimization of the effects of line broadening by the finite lifetime of the transitions and experimental broadening contributions.

The changes in $B$ being a measure for the amplitude of the angular part of the spin-orbit operator showing a $12 \%$ decrease with increasing spacer layer thickness which further partially corroborates with the increasing Co valency or changes of the local magnetic moment. Theoretical and experimental studies have shown that the ratio of a $3 d$ transition metal atom generally increases with its magnetic moment. However, a clear relation, or a sum rule, relating these two quantities has not been established, and the absolute value of the magnetic moment cannot be obtained directly from this ratio. ${ }^{50}$ Recently, $\mathrm{XMCD}$ spectra from $\mathrm{Co}-\mathrm{Au}$ multilayers have demonstrated that changes in the neighborhood of the Co atoms can suppress its magnetism due to impurities and interdiffusion. ${ }^{51}$ The increased thickness of the Au layers might have lead to an enhanced concentration of Au impurities around the Co atoms. The reduced magnetization of Co with the increasing thickness of the spacer layer is in agreement with the PPMS [see Figs. 3(a) and 3(b)] and PNR measurements [see Figs. 12(a), 12(b), and 13].

\section{E. Interface magnetism}

Furthermore, we discuss the various possibilities that can be responsible for the observed magnetic behavior with spacer thickness such as (a) exchange coupling across the spacer, (b) interfacial dilution, and (c) perpendicular anisotropy.

a. Exchange coupling. In the present scenario, the unusual thickness dependence of the spacer layer on exchange bias therefore raises the question of whether there is RKKY type coupling or magnetostatic coupling or any other mechanism that might determine the enhanced exchange coupling, besides the variations in relative proportion of AF-FM. In magnetic multilayers, magnetic moments can be looked upon as immersed in a sea of the conduction electrons of the spacer layer which gives rise to damped long-range oscillation of the interlayer exchange coupling as a function of the spacer thickness. ${ }^{52,53}$ The magnetostatic interaction between two FM films, separated by a nonmagnetic spacer, is caused by the stray fields (magnetostatic coupling) with antiparallel magnetizations. However, following Néel's theory (in presence of a correlated roughness), an interlayer coupling can be induced that is ferromagnetic in nature and decreases exponentially. ${ }^{54}$ In the possible coupling mechanisms discussed above, the coupling strength definitively dampens down at around $2.0-5.0 \mathrm{~nm}$ of spacer thickness, again ruling out such a possibility in our case.

Possible long-range interaction across a spacer layer is common in magnetic multilayers. For example, Gierlings et al. ${ }^{13}$ investigated the effect of a Au spacer across a similar $\mathrm{Co}-\mathrm{CoO}$ system where they could find induced magnetic moments in Au by local $s-d$ hybridization with the $d$ band of the nearest Co atoms. A canted magnetic structure in the film plane, thus realized at the interface across a Au spacer of $\leqslant 1.0 \mathrm{~nm}$, reduces the exchange coupling. Very recently, Meng et al. ${ }^{17}$ and Valev et al. ${ }^{55}$ have reported an interlayer coupling between $\mathrm{CoO}$ and $\mathrm{Fe}$ separated by at least $4.0 \mathrm{~nm}$ (10 monolayers) of $\mathrm{Ag}$ and $3.5 \mathrm{~nm}$ of $\mathrm{Cu}$ spacer layer, respectively. The pinning centers deep inside the AF layer, contributing to the exchange bias field are also indicative of the long range aspect of it. ${ }^{56}$ Note that in our case, the thickness of the Au spacers are at least an order of magnitude larger. Moreover, PNR shows that there is no in-plane canted spin structure. This may rule out spin-canting due to possible pin-hole formation between the two consecutive $\mathrm{Co}-\mathrm{CoO}$ layers on either side of the Au layer.

b. Interfacial dilution. In this regard, it is natural to think of interfacial dilution for a magnetic/nonmagnetic interface. In an earlier case, a decrease of the thermal stability of the 
AF was conjectured. ${ }^{14}$ It was shown that the bias field can be slightly increased (only by around $100 \mathrm{Oe}$ ) by $\mathrm{Cu}$ dilution in IrMn based exchange biased system. Moreover, such a dilution effect affects the blocking temperature of the system as well. In the present case, we can rule out any dilution effect as we do not observe any significant change in the blocking temperatures for our multilayers. We may also rule out diffusion of $\mathrm{Au}$ impurities into the $\mathrm{Co}$ and $\mathrm{CoO}$ layers as $\mathrm{Co}$ and $\mathrm{Au}$ are immiscible (positive heat of mixing) at or below RT and we see no magnetic dead layers by PNR.

c. Perpendicular magnetic anisotropy. In ultrathin films, perpendicular magnetic anisotropy (PMA) effects may commonly result from interface and/or magnetoelastic effects apart from more intrinsic magnetocrystalline anisotropy. Magnetoelasticity is dominant with decreasing film thickness which can make PMA restricted to low thicknesses (typically $1 \mathrm{~nm}$ ). Stöhr has shown that the orbital moment on a Co atom becomes anisotropic (below 10 monolayers or $\sim 2.5 \mathrm{~nm}$ ) through quenching effects by the anisotropic ligand fields of the neighboring $\mathrm{Au}$ atoms (which can be as thick as $28.0 \mathrm{~nm}) .{ }^{47}$ Recently, Paul et al. have also observed strong PMA in $[\mathrm{Co}(2.0 \mathrm{~nm}) / \mathrm{Au}(2.0 \mathrm{~nm})]_{32}$ multilayers. Note that the Co layer thicknesses were restricted to 8 monolayers instead of usual range of 1-2 monolayers. ${ }^{57}$

The thickness of the Co layers in the present case are $11.0 \mathrm{~nm}(\sim 44 \mathrm{ML})$ thus one should not expect any PMA in this range. However, for the Co_50 ML, as compared to the Co_25 ML, we find $\approx 13 \%$ decrease in the magnetic moment (when measured along the sample plane, either by PPMS, PNR, or $L$-edge spectroscopy). The reduced magnetic moment indicates that there can be a relative increase in PMA. Schematics of possible scenarios for the magnetic structures of the layers are shown in Fig. 17. As an example, we have shown the cases for two the MLs namely, Co_25 and Co_50 at the FM- interfaces. From the $K$-edge spectroscopy, we observe the following. (i) For FM(Co)-Au interfaces: $\approx 30 \%$ decrease in the proportion of Co thickness (increase in the Au layer thickness can affect the interface with the Co layer). This will reduce the effective magnetic Co thickness from $11.0 \mathrm{~nm}$ to around $8.0 \mathrm{~nm}$. Such a decrease can be due to canting of the Co moments at the interfaces. This would then obviously increase the probability of PMA. (ii) For $\mathrm{AF}\left(\mathrm{CoO}+\mathrm{Co}_{3} \mathrm{O}_{4}\right)$ -

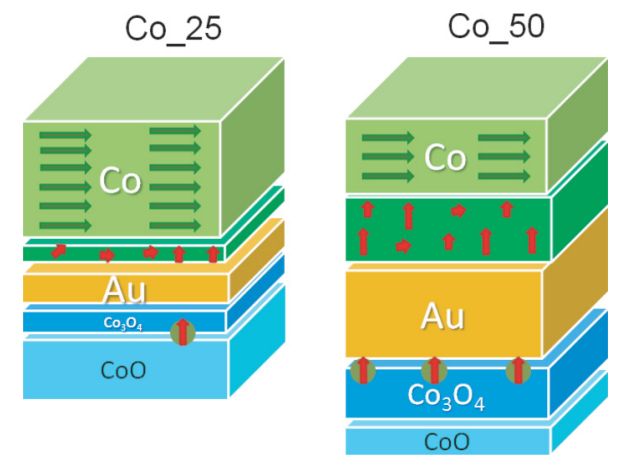

FIG. 17. (Color online) The schematics of the layer structures at the Co-Au and $\mathrm{Au}-\mathrm{CoO}$ interfaces of the two MLs Co_25 and Co_50. The arrows represent the out-of-plane FM spins (red) and the in-plane FM spins (green).
$\mathrm{Au}$ interfaces: $\approx 4 \%$ increase of the $\mathrm{Co}_{3} \mathrm{O}_{4}$ proportion within the AF layers. This can, on the other hand, increase the number of uncompensated spins within the AF. On the other hand, they can have increased un-oxidized proportions of $\mathrm{Co}\left(\mathrm{Co}_{x} \mathrm{O}_{y}\right)$. Since the absolute thickness of such an unoxidized layer is very small (below 10 monolayers), with increased possibility of $\mathrm{Au}$ at its neighborhood, the unoxidized Co magnetic moments can turn out of plane. Thus one can argue that the observed increase in the bias field can be attributed to the canting of the Co moments at the Co-Au interface (effective reduction in the FM layer thickness) and/or increased proportion of uncompensated moments within the $\mathrm{CoO}$ layers.

Presumably, the uncompensated AF moments within the $\mathrm{CoO}$ are located at approximately $1-2 \mathrm{~nm}$ from the interface and a canting of those spins would have reduced the bias field as a result of net reduction in the number of uncompensated spins. Similarly, with an induced magnetism in $\mathrm{Au}$ at the $\mathrm{Co}-\mathrm{Au}$ (FM-Au) interface, there would have been a decrease in the bias field (effective increase of the FM layer). On the other hand, an induced magnetism within the AF, adjacent to a FM (AF-FM interface), can only reduce the bias field rather than increasing it. ${ }^{58}$ However, the effect of an induced magnetism at the $\mathrm{CoO}-\mathrm{Au}$ (AF-Au interface) interface would have been interesting to investigate. Thus we can rule out any induced moment either at the FM-Au or AF-FM interfaces or canting of the AF moments.

Paul et al. have recently reported that with the application of a perpendicular cooling field (perpendicular to the film plane) one can induce an exchange bias in $\mathrm{Co} / \mathrm{CoO} / \mathrm{Au} \mathrm{MLs}$ which is directed out of plane. ${ }^{20}$ This unconventional exchange biasing was possible mainly due to the difference in uniaxial anisotropy energies of the $\mathrm{Co}\left(\sim 5 \times 10^{5} \mathrm{erg} \mathrm{cm}^{-3}\right)^{59}$ and the $\mathrm{CoO}\left(\sim 25 \times 10^{7} \mathrm{erg} \mathrm{cm}^{-3}\right)^{60}$ layer apart from the possible intrinsic tendency of PMA at the Co-Au interfaces. In the present context, we performed similar measurements on our MLs. An increasing tendency of induced bias in the out-ofplane direction would essentially confirm the increasing outof-plane canted proportion of the Co moments, with increasing spacer thickness. In other words, the larger the number of outof-plane uncompensated moments is the larger the reduction of the moments in the film-plane will be.

Figure 18 shows the longitudinal magnetization measured at $10 \mathrm{~K}$ for an out-of-plane cooling field $\left(H_{\mathrm{FC}}=+4.0 \mathrm{kOe}\right)$

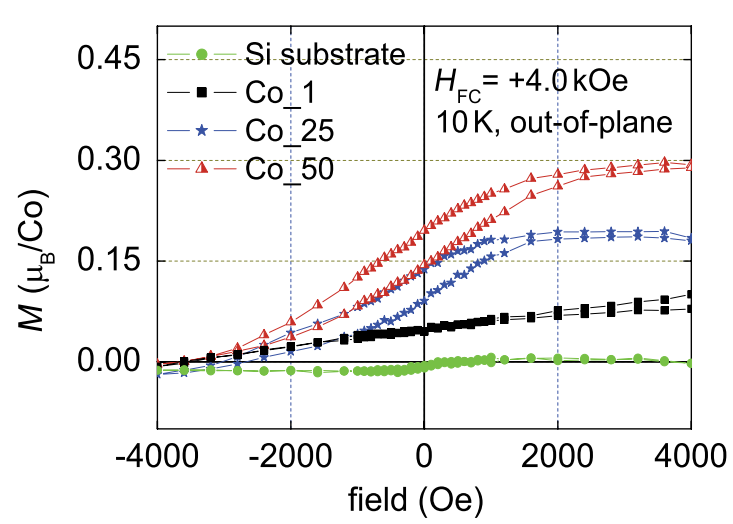

FIG. 18. (Color online) Magnetization loops for the substrate, Co_1, Co_25, and Co_50 MLs for out-of-plane cooling field. 
for the Co_1, Co_25, and C_50 MLs. The signal can be compared with the background signal from a Si substrate measured with the same conditions in the PPMS, showing a typical linear paramagnetic slope. One may note that a hysteresis (opening up of the loop) is seen only for the Co_25 and Co_50 MLs and not for the Co_1 ML. This is expected since the Co_1 ML does not contain any Au spacer layer. This obviously indicates the increased tendency of PMA with increased spacer layer thickness. Out-of-plane canting of the Co moments have resulted in the net reduction in the moments in the film-plane. Additionally, we find a distinct but small vertical shift of the hysteresis loops for all of our MLs (and not for the substrate). Vertical shifts are related to uncompensated moments at the FM-AF interfaces or noncollinear magnetic structure at interfaces. ${ }^{61}$ Depending upon their origin (which, however, remains unclear) that can be in the $\mathrm{AF}$ and/or in the FM, they can be, in principle, correlated or uncorrelated to the $H_{\mathrm{eb}}$ values. Thus nonmagnetic spacers are shown to affect the interface magnetism without changing the microstructure.

Canting of the Co moments or induced magnetism of the $\mathrm{Au}$ layer can be looked upon as due to $s-d$ hybridization. However, whether the hybridization is at the Co-Au interface or at the $\mathrm{CoO}-\mathrm{Au}$ interface is beyond the scope of the available techniques. We suggest that a deeper insight into the impact of the $\mathrm{AF} / \mathrm{Au}$ or $\mathrm{FM} / \mathrm{Au}$ interface magnetism including the effects of roughness and interdiffusion on the exchange bias phenomena has to be undertaken for a better understanding.

\section{CONCLUSION}

We observe a systematic increase in the exchange bias fields and the coercive fields with increasing thicknesses of the $\mathrm{Au}$ layer that are immersed between the $\mathrm{Co} / \mathrm{CoO}$ bilayers which may be an important route to improve future devices using the exchange bias. The structural evolution of the ferromagnetic grains as seen by XTEM measurements is interrupted by growing Au layers of appropriate thickness. The grains in the Au layers are of the order of the Au layer thickness. The $\mathrm{Au}$ layer decouples the structural and magnetic properties of the magnetic bilayers thus inhibiting the evolution of domains across the heterostructure. Evidence of this is provided by off-specular polarized neutron scattering. Interestingly, the magnetic moment per atom in the FM layers is seen to decrease with increasing thickness of the $\mathrm{Au}$ spacer layer. This is confirmed by PPMS and PNR measurements. Subloop shifts of the hysteresis around the blocking temperature indicates a different initial AF-FM domain configuration for samples with $\mathrm{Au}$ spacers (as compared to that without spacers).

The increase in the bias field, to some extent, accounts for the relative proportions of the FM and AF species as inferred from the XANES and the XAS measurements. However, a larger extent of the increment is owed to reduced magnetic moment of the Co layer as inferred from the magnetometry and PNR measurements. Such a reduction is plausibly owed to the out-of-plane orientation tendencies of the Co moments at the $\mathrm{Co}-\mathrm{Au}$ interfaces. By performing perpendicular field cooling, we could demonstrate an increasing tendency of the Co moments to orient out-of-plane which effectively explains the in-plane decrease of the magnetic moment with increased Au spacer thickness. Perpendicular field cooling is thus seen as a novel way to characterize the uncompensated spins at the interface of such exchange coupled systems. Further detail on the interfaces, for example, hybridization at the AF/NM and $\mathrm{FM} / \mathrm{NM}$ interfaces and changes in the AF-FM domain configurations (due to the NM) can be topics for future investigations.

\section{ACKNOWLEDGMENTS}

This work was supported by the Deutsche Forschungsgemeinschaft via the Transregional Collaborative Research Centers TRR 80. We would also like to thank Ulrike Bloeck and Andreas Bauer for the assistance in TEM and PPMS measurements, respectively.
*Corresponding author: amitesh.paul@frm2.tum.de

${ }^{1}$ A. Moser, K. Takano, D. T. Margulies, M. Albrecht, Y. Sonobe, Y. Ikeda, S. Sun, and E. E. Fullerton, J. Phys. D 35, R157 (2002); D. Weller and A. Moser, IEEE Trans. Magn. 35, 4423 (1999).

${ }^{2}$ W. H. Meiklejohn and C. P. Bean, Phys. Rev. 102, 1413 (1956).

${ }^{3}$ M. G. Blamire, M. Ali, C.-W. Leumg, C. H. Marrows, and B. J. Hickey, Phys. Rev. Lett. 98, 21702 (2007).

${ }^{4}$ J. Nogués and I. K. Schuller, J. Magn. Magn. Mater. 192, 203 (1999); A. E. Berkowitz and K. Takano, ibid. 200, 552 (1999).

${ }^{5}$ M. Gierlings, M. J. Prandolini, H. Fritzsche, M. Gruyters, and D. Riegel, Phys. Rev. B 65, 092407 (2002).

${ }^{6}$ A. Paul, C. M. Schneider, and J. Stahn, Phys. Rev. B 76, 184424 (2007).

${ }^{7}$ M. D. Stiles and R. D. McMichael, Phys. Rev. B 59, 3722 (1999).

${ }^{8}$ M. Gruyters and D. Schmitz, Phys. Rev. Lett. 100, 077205 (2008).

${ }^{9}$ M. R. Fitzsimmons, P. Yashar, C. Leighton, I. K. Schuller, J. Nogues, C. F. Majkrzak, and J. A. Dura, Phys. Rev. Lett. 84, 3986 (2000).
${ }^{10}$ A. Paul, E. Kentzinger, U. Rücker, and T. Bruckel, Phys. Rev. B 74, 054424 (2006).

${ }^{11}$ A. Paul and S. Mattauch, Appl. Phys. Lett. 95, 092502 (2009).

${ }^{12}$ A. Paul, E. Kentzinger, U. Rücker, D. E. Bürgler, and P. Grünberg, Phys. Rev. B 70, 224410 (2004).

${ }^{13}$ M. Gierlings, M. J. Prandolini, M. Gruyters, T. Funk, D. Riegel, and W. D. Brewer, Eur. Phys. J. B 45, 137 (2005).

${ }^{14}$ M. Fecioru-Morariu, S. R. Ali, C. Papusoi, M. Sperlich, and G. Güntherodt, Phys. Rev. Lett. 99, 097206 (2007).

${ }^{15}$ M. J. Prandolini, Rep. Prog. Phys. 69, 1235 (2006).

${ }^{16}$ S. Laureti, S. Y. Suck, H. Haas, E. Prestat, O. Bourgeois, and D. Givord, Phys. Rev. Lett. 108, 077205 (2012).

${ }^{17}$ Y. Meng, J. Li, P.-A. Glans, C. A. Jenkins, E. Arenholz, A. Tan, J. Gibbons, J. S. Park, C. Hwang, H. W. Zhao, and Z. Q. Qiu, Phys. Rev. B 85, 014425 (2012).

${ }^{18}$ A. N. Dobrynin and D. Givord, Phys. Rev. B 85, 014413 (2012).

${ }^{19}$ S. R. Ali, M. R. Ghadimi, M. Fecioru-Morariu, B. Beschoten, and

G. Guntherodt, Phys. Rev. B 85, 012404 (2012).

${ }^{20}$ A. Paul, N. Paul, and S. Mattauch, New J. Phys. 13, 063009 (2011). 
${ }^{21}$ A. Paul, D. Bürgler, M. Luysberg, and P. Grünberg, Europhys. Lett. 68, 233 (2004).

${ }^{22}$ C. Leighton, J. Nogués, B. J. Jönsson-Åkerman, and Ivan K. Schuller, Phys. Rev. Lett. 84, 3466 (2000).

${ }^{23}$ A. Paul, Pramana: J. Phys. 78, 1 (2012).

${ }^{24}$ U. Nowak, K. D. Usadel, J. Keller, P. Miltenyi, B. Beschoten, and G. Güntherodt, Phys. Rev. B 66, 014430 (2002).

${ }^{25}$ Y. Zhao, T. E. Feltes, J. R. Regalbuto, R. J. Mayer, and R. F. Klie, J. Appl. Phys. 108, 063704 (2010).

${ }^{26}$ F. Wilhelm, M. Angelakeris, N. Jaouen, P. Poulopoulos, E. Th. Papaioannou, Ch. Mueller, P. Fumagalli, A. Rogalev, and N. K. Flevaris, Phys. Rev. B 69, 220404(R) (2004).

${ }^{27}$ D. Mauri, E. Kay, D. Scholl, J. Kent, and J. Kent Howard, J. Appl. Phys. 62, 2929 (1987).

${ }^{28}$ E. Girgis, R. D. Portugal, H. Loosvelt, M. J. Van Bael, I. Gordon, M. Malfait, K. Temst, C. Van Haesendonck, L. H. A. Leunissen, and R. Jonckheere, Phys. Rev. Lett. 91, 187202 (2003).

${ }^{29}$ K. O'Grady, L. E. Fernandez-Outon, and G. Vallejo-Fernandez, J. Magn. Magn. Mater. 322, 883 (2010).

${ }^{30}$ G. Herzer, J. Magn. Magn. Mater. 294, 99 (2005).

${ }^{31}$ J. Geshev, L. G. Pereira, and J. E. Schmidt, Phys. Rev. B 66, 134432 (2002).

${ }^{32}$ A. Paul, E. Kentzinger, U. Rücker, and Th. Brückel, Phys. Rev. B 73, 092410 (2006).

${ }^{33}$ A. R. Modak, David J. Smith, and S. S. P. Parkin, Phys. Rev. B 50, 4232 (1994).

${ }^{34}$ D. H. Wei, S. C. Chou, T. S. Chin, C. C. Yu, Y. Liou, and Y. D. Yao, J. Appl. Phys. 97, 10N121 (2005).

${ }^{35}$ A. Ney, P. Poulopoulos, M. Farle, and K. Baberschke, Phys. Rev. B 62, 11336 (2000)

${ }^{36}$ C. F. Majkrzak, J. D. Axe, and P. Böni, J. Appl. Phys. 57, 3657 (1985); J. F. Ankner, C. F. Majkrzak, and H. Homma, ibid. 73, 6436 (1993).

${ }^{37}$ J. Orna, L. Morellón, P. A. Algarabel, J. M. De Teresa, A. Fernández-Pacheco, G. Simón, C. Magen, J. A. Pardo, and M. R. Ibarra, Advances Science Technology 67, 82 (2010).

${ }^{38}$ B. P. Toperverg, in Polarized Neutron Scattering, Matter and Materials Vol. 12 (Forschungszentrum Jülich, Germany, 2002), p. 247; H. Zabel et al. in Handbook of Magnetism and Advanced Magnetic Materials, edited by H. Kronmuller and S. Parkin (Wiley, NY, 2007), p. 1237.

${ }^{39}$ A. Paul and S. Mattauch, J. Appl. Phys. 108, 053918 (2010).
${ }^{40}$ Jörg F. Löffler, Werner Wagner, and Gernot Kostorz, J. Appl. Crystallogr. 33, 451 (2000).

${ }^{41}$ J. F. Löffler, H. B. Braun, and W. Wagner, J. Appl. Phys. 85, 5187 (1999).

${ }^{42}$ B. Ravel and M. Newville, J. Synchrotron Radiat. 12, 537 (2005).

${ }^{43}$ A. L. Ankudinov, B. Ravel, J. J. Rehr, and S. D. Conradson, Phys. Rev. B 58, 7565 (1998).

${ }^{44}$ S. Gangopadhyay, G. C. Hadjipanayis, C. M. Sorensen, and K. J. Klabunde, J. Appl. Phys. 73, 6964 (1993).

${ }^{45}$ X. Y. Lang, W. T. Zheng, and Q. Jiang, Nanotechnology 18, 155701 (2007).

${ }^{46}$ J. A. Borchers, Y. Ijiri, D. M. Lind, P. G. Ivanov, R. W. Erwin, Aron Qasba, S. H. Lee, K. V. O’Donovan, and D. C. Dender, Appl. Phys. Lett. 77, 4187 (2000).

${ }^{47}$ J. Stöhr, J. Magn. Magn. Mater. 200, 470 (1999).

${ }^{48}$ J. T. Lau, J. Rittmann, V. Zamudio-Bayer, M. Vogel, K. Hirsch, Ph. Klar, F. Lofink, T. Mller, and B. V. Issendorff, Phys. Rev. Lett. 101, 153401 (2008)

${ }^{49}$ B. T. Thole and G. vander Laan, Phys. Rev. B 38, 3158 (1988).

${ }^{50}$ M. Koshinoa, H. Kuratab, S. Isodaa, and T. Kobayashia, Micron 31, 373 (2000).

${ }^{51}$ O. Šipr, J. Minár, J. Vackář, and H. Ebert, Phys. Rev. B 75, 134422 (2007).

${ }^{52}$ P. Grünberg, R. Schreiber, Y. Pang, M. B. Brodsky, and H. Sowers, Phys. Rev. Lett. 57, 2442 (1986).

${ }^{53}$ N. N. Shukla, A. Sen, and R. Prasad, Phys. Rev. B 76, 174417 (2007).

${ }^{54}$ J. Moritz, F. Garcia, J. C. Toussaint, B. Dieny, and J. P. Nozières, Europhys. Lett. 65, 123 (2004).

${ }^{55}$ V. K. Valev, M. Gruyters, A. Kirilyuk, and Th. Rasing, Phys. Rev. Lett. 96, 067206 (2006).

${ }^{56}$ R. Morales, Zhi-Pan Li, J. Olamit, Kai Liu, J. M. Alameda, and Ivan K. Schuller, Phys. Rev. Lett. 102, 097201 (2009).

${ }^{57}$ A. Paul and P. Böni (unpublished).

${ }^{58}$ A. Paul, Appl. Phys. Lett. 97, 032505 (2010).

${ }^{59}$ W. Wulfhekel, S. Knappmann, and H. P. Oepen, J. Appl. Phys. 79, 988 (1996).

${ }^{60}$ W. L. Roth, Phys. Rev. 110, 1333 (1958).

${ }^{61}$ E. C. Passamania, C. Laricaa, C. Marquesa, J. R. Provetia, A. Y. Takeuchib, and F. H. Sanchez, J. Magn. Magn. Mater. 299, 11 (2006). 